\title{
gु \\ Quantum simulation of materials at micron scales and beyond
}

\author{
Qing Peng, ${ }^{1}$ Xu Zhang, ${ }^{1}$ Linda Hung, ${ }^{2}$ Emily A. Carter, ${ }^{3}$ and Gang Lu ${ }^{1, *}$ \\ ${ }^{1}$ Department of Physics and Astronomy, California State University Northridge, Northridge, California, USA \\ ${ }^{2}$ Program in Applied and Computational Mathematics, Princeton University, Princeton, New Jersey, USA \\ ${ }^{3}$ Department of Mechanical and Aerospace Engineering, Princeton University, Princeton, New Jersey, USA
}

(Received 14 April 2008; revised manuscript received 4 June 2008; published 21 August 2008)

\begin{abstract}
We present a multiscale modeling approach that can simulate multimillion atoms effectively via densityfunctional theory. The method is based on the framework of the quasicontinuum (QC) approach with orbitalfree density-functional theory (OFDFT) as its sole energetics formulation. The local QC part is formulated by the Cauchy-Born hypothesis with OFDFT calculations for strain energy and stress. The nonlocal QC part is treated by an OFDFT-based embedding approach, which couples OFDFT nonlocal atoms to local region atoms. The method-QCDFT - is applied to a nanoindentation study of an Al thin film, and the results are compared to a conventional QC approach. The results suggest that QCDFT represents a new direction for the quantum simulation of materials at length scales that are relevant to experiments.
\end{abstract}

DOI: 10.1103/PhysRevB.78.054118

PACS number(s): 71.15.Mb, 62.20.-x, 71.15.Dx

\section{INTRODUCTION}

The ability to perform quantum simulations of materials properties over length scales that are relevant to experiments represents a grand challenge in computational materials science. If one could treat multimillions or billions of electrons effectively at micron scales, such first-principles quantum simulations could revolutionize materials research and pave the way to the computational design of advanced materials. There are two principal reasons why quantum simulations at relevant experimental scales are important. First of all, it allows a direct comparison between theory and experiment. For example, the rapidly emerging field of nanotechnology demands realistic and accurate modeling of material systems at the nanoscale, including nanoparticles, nanowires, quantum dots, nanoelectromechanical systems (NEMS), and microelectromechanical systems (MEMS). All these nanosystems could reach a length scale of microns and contain millions or billions of electrons, if not more. Second, quantum simulations at larger scales are essential even for extended bulk crystals where periodic boundary conditions may be used. This is due to the fact that a real bulk solid always contains lattice defects (or impurities) whose interactions are long range-dislocations being the prominent example. An insufficiently large periodic unit cell would lead to unrealistically high concentrations of defects and/or impurities, rendering the results of such simulations questionable.

In this paper, we propose a multiscale approach that is based entirely on density-functional theory (DFT) and allows quantum simulations at the micron scale and beyond. The method, termed QCDFT, combines the coarse-graining idea of the quasicontinuum (QC) approach and the coupling strategy of the quantum mechanics/molecular mechanics (QM/ MM) method, and represents a potentially major advance in the quantum simulation of materials properties. It should be stated at the outset that QCDFT is not a brute-force electronic structure method, but rather a multiscale approach that can treat large systems-effectively up to billions of electrons. Therefore, some of the electronic degrees of freedom are reduced to continuum degrees of freedom in QCDFT. On the other hand, although QCDFT utilizes the idea of QM/MM coupling, it does not involve any classical/ empirical potentials (or force fields) in the formulation-the energy calculation of QCDFT is entirely based on orbitalfree DFT (OFDFT). This is an important feature and advantage of QCDFT, which qualifies it as a bona fide quantum simulation method.

Since QCDFT is formulated within the framework of the QC method, we shall give an introduction to QC in the context of the multiscale modeling of materials in Sec. II A. Then in Sec. II B, we introduce the OFDFT formalism, an approximation to Kohn-Sham (KS) DFT that offers a necessary compromise between computational accuracy and efficiency. In Sec. II C, we explain how OFDFT is used to evaluate energy and stress in the local QC region. In Sec. II D, we discuss the necessity of employing the QM/MM scheme and introduce an OFDFT-based QM/MM approach that can treat the nonlocal QC region accurately and efficiently. In Sec. II E, we propose an approach to address an important issue in the QC method-the ghost force. The parallelization of the QCDFT method is briefly described in Sec. II F. In Sec. II G, we compare the QCDFT method to other related approaches. After the QCDFT method is introduced, we apply it to the study of nanoindentation of an $\mathrm{Al}$ thin film. The introduction of nanoindentation and the model setup is presented in Sec. III A. In Secs. III B and III C, we discuss the loading procedure and relevant parameters used in the QCDFT calculations. We present the nanoindentation results in Sec. IV and finally our conclusions in Sec. V.

\section{QCDFT METHODOLOGY}

The QCDFT method is based on the quasicontinuum framework with an OFDFT energy formulation. For completeness, we provide in this section a detailed description of various components of QCDFT, although some of them have been published previously.

\section{A. Quasicontinuum method}

The quasicontinuum $(\mathrm{QC})$ method $^{1,2}$ is a concurrent multiscale approach ${ }^{3}$ that combines atomistic models with con- 
tinuum theories, and thus offers an advantage over conventional atomistic simulations in terms of computational efficiency. The idea underlying the QC method is that atomistic processes of interest often occur in very small spatial domains while the vast majority of atoms in the material behave according to well-established continuum theories. To exploit this fact, the QC method retains atomic resolution only where necessary and coarsens to a continuum finite element description elsewhere. The original formulation of QC was limited to classical potentials for describing interactions between atoms. However, since many materials properties depend crucially on the behavior of electrons, such as bond breaking/forming at crack tips or defect cores, chemical reactions with impurities, surface reactions and reconstructions, electron excitation and magnetism, etc., it is desirable to incorporate appropriate quantum mechanical descriptions into the QC formalism. QCDFT is one strategy to fill this role.

The goal of the QC method is to model an atomistic system without explicitly treating every atom in the problem. ${ }^{1,2}$ This is achieved by replacing the full set of $N$ atoms with a small subset of $N_{r}$ "representative atoms" or repatoms $\left(N_{r}\right.$ $\ll N$ ) that approximate the total energy through appropriate weighting functions. The weighting functions can be physically interpreted as the number of atoms represented by each repatom. The weighting functions depend on the local deformation state and are determined on-the-fly in QC simulations. The energies of individual repatoms are computed in two different ways depending on the deformation in their immediate vicinity. Atoms experiencing large variations in the deformation gradient field on an atomic scale are computed in the same way as in a standard atomistic method. In QC these atoms are called nonlocal atoms to reflect the fact that their energy depends on the positions of their neighbors in addition to their own position. In contrast, the energies of atoms experiencing a smooth deformation field on the atomic scale are computed based on the deformation gradient $\{\mathbf{G}\}$ in their vicinity as befitting a continuum model. These atoms are called local atoms because their energy is based only on the deformation gradient at the point where it is computed. In a classical system, the total energy $E_{\text {tot }}$ can be written as $E_{\mathrm{tot}}=\sum_{i=1}^{N} E_{i}$ (with $E_{i}$ the energy of atom $i$ ), and is approximated as

$$
E_{\mathrm{tot}}^{\mathrm{QC}}(\{\mathbf{R}\},\{\mathbf{G}\})=\sum_{i=1}^{N^{\mathrm{nl}}} E_{i}(\{\mathbf{R}\})+\sum_{j=1}^{N^{\mathrm{loc}}} n_{j} E_{j}^{\mathrm{loc}}(\{\mathbf{G}\}) .
$$

The first term is an atomistic region of $N^{\mathrm{nl}}$ nonlocal atoms with energy dependent upon the coordinates of neighbor repatoms at $\{\mathbf{R}\}$ as in the usual atomistic fashion. The second term gives the contribution from local repatoms each of which represents $n_{j}$ physical atoms with energy dependent upon local deformation gradients $\{\mathbf{G}\}$ characterizing the finite strain around its position. The basic assumption employed is the Cauchy-Born rule, which relates the continuum deformation at a point to the motion of the atoms in the underlying lattice represented by this point.

In QCDFT, the calculations of $E_{j}^{\mathrm{loc}}(\{\mathbf{G}\})$ and stress in the continuum regions are based on OFDFT, which is the same energy formulation used in the nonlocal atomistic region. This makes the passage from the atomistic to continuum regions seamless since the same underlying material description is used in both. This seamless description enables the model to adapt automatically to changing circumstances (e.g., the nucleation of new defects or the migration of existing defects). This adaptability is difficult for many other multiscale methods and is one of the QC method's main strengths. On the other hand, a consequence of the partitioning into local and nonlocal regions and the existence of a well-defined total energy for the entire system is the presence of nonphysical ghost forces at the interface. These can be eliminated by self-consistent application of dead load corrections. $^{2}$

In the following, we outline the energy and force formulations for both local and nonlocal regions of QCDFT. In the local region, a finite element mesh is constructed with each repatom on the vertex of several surrounding finite elements. The energy and force of each local repatom can be obtained from the strain energy density and the stress tensor of the finite elements that share the same repatom. More specifically, according to the Cauchy-Born rule, the deformation gradient $\mathbf{G}$ is uniform within a finite element, therefore the local energy density $\varepsilon$ and the stress tensor for each finite element can be calculated as a perfect infinite crystal undergoing a uniform deformation specified by G. In other words, one could perform an OFDFT-based energy/stress calculation for an infinite crystal by using periodic boundary conditions with the primitive lattice vectors of the deformed crystal, $\mathbf{h}_{i}$, given by

$$
\mathbf{h}_{i}=\mathbf{G H}_{i}, \quad i=1,2,3 .
$$

Here $\mathbf{H}_{i}$ are the primitive lattice vectors of the undeformed crystal and the volume of the primitive unit cell is $\Omega_{0}$. The details of the OFDFT calculation can be found in Sec. II C. Once the strain energy density $\varepsilon\left(\mathbf{G}_{k}\right)$ is determined, the energy contribution of the $j$ th local repatom is given as

$$
E_{j}^{\mathrm{loc}}(\{\mathbf{G}\})=\sum_{k=1}^{M_{j}} w_{k} \varepsilon\left(\mathbf{G}_{k}\right) \Omega_{0},
$$

where $M_{j}$ is the total number of finite elements represented by the $j$ th repatom, and $w_{k}$ is the weight assigned to the $k$ th finite element. The force on the $j$ th local repatom is defined as the gradient of the total energy with respect to its coordinate $\mathbf{R}_{j}^{\text {loc }}$. In practice, the nodal force on each finite element is calculated from the stress tensor of the finite element by using the principle of virtual work. ${ }^{4}$ The force on the repatom is then obtained by summing the nodal force contributions from each surrounding finite element.

For the energy/force calculation in the nonlocal region, we resort to a QM/MM approach that was developed recently for metals. ${ }^{5}$ The coupling between the QM and MM regions is achieved quantum mechanically within an OFDFT formulation. Although the detailed implementation of the $\mathrm{QM} / \mathrm{MM}$ approach is presented in Sec. II D, we wish to stress two important points here: (i) The original QC formulation assumes that the total energy can be written as a sum over individual atomic energies. This condition is not satis- 
fied by quantum mechanical models. The energy of the nonlocal region is now a functional of total electron density, so instead of the expression in Eq. (1), the total energy of QCDFT should be expressed as

$$
E_{\mathrm{tot}}^{\mathrm{QCDFT}}(\{\mathbf{R}\},\{\mathbf{G}\})=E^{\mathrm{nl}}\left[\rho^{\mathrm{tot}}\right]+\sum_{j=1}^{N^{\mathrm{loc}}} n_{j} E_{j}^{\mathrm{loc}}(\{\mathbf{G}\}) .
$$

Here $\rho^{\text {tot }}$ is the total electron density in the nonlocal region as well as the coupling nonlocal/local region, i.e., the buffer region in the following discussion. (ii) The nonlocal energy, $E^{\mathrm{nl}}$, should be calculated with appropriate boundary conditions; that is to say, it should include the interaction energy between the nonlocal atoms and neighboring local atoms. In the original QC framework, this requirement is realized by including dummy atoms in the energy/force calculation of a given nonlocal repatom. These dummy atoms are in the local region and within the cut-off radius of the given nonlocal repatom. The dummy atoms are not independent degrees of freedom in the local region, but rather slaves to the local repatoms. In this way, the nonlocal calculation is carried out with the appropriate boundary conditions, and at the same time, the energy of the dummy atoms is still treated with the Cauchy-Born rule, consistent with their status. In the QCDFT approach, a buffer region including the dummy atoms and local repatoms that are adjacent to the nonlocal repatoms is selected as the "MM" region, and the nonlocal atoms constitute the QM region. The nonlocal atoms are treated by OFDFT, and the coupling between the "MM" and $\mathrm{QM}$ region is also formulated within OFDFT. Therefore the entire system is formulated with one energy functional, OFDFT, which is evaluated at two levels of approximation depending upon the inhomogeneity or homogeneity of the local deformation. Note that "MM" here is actually a misnomer: The local atoms are treated by OFDFT with the Cauchy-Born rule as mentioned earlier, and we retain the designation "MM" solely to indicate the similarity to the earlier coupling scheme. ${ }^{5}$

\section{B. OFDFT formulation}

OFDFT is the energy formulation of choice for the present QCDFT method, although KS DFT can also be used for calculating the local and nonlocal energies (see Sec. II G). OFDFT is an efficient implementation of densityfunctional theory which approximates the kinetic energy of noninteracting electrons in terms of their density, instead of the KS orbitals. ${ }^{6-11}$ In doing so, OFDFT reduces the degrees of freedom from $3 N$ to 3 ( $N$ being the number of electrons). It also removes the need for expensive orthonormalization and $k$-point sampling associated with the KS orbitals. The resulting $\mathcal{O}(N \ln N)$ scaling of the calculation allows the study of systems larger than $10^{5}$ atoms when periodic boundary conditions are used. ${ }^{12}$

In OFDFT, the total energy is expressed as an explicit functional of electron density $\rho(\mathbf{r})$ :

$$
E_{\mathrm{OF}}[\rho]=T_{\mathrm{s}}[\rho]+E_{\mathrm{H}}[\rho]+E_{\mathrm{e}-\mathrm{i}}[\rho]+E_{\mathrm{xc}}[\rho]+E_{\mathrm{i}-\mathrm{i}} .
$$

The various terms in Eq. (5) represent the noninteracting electronic kinetic energy, the Hartree electron repulsion en- ergy, the electron-ion attraction energy, the electron exchange-correlation energy, and the ion-ion repulsion energy, respectively.

The lack of wave functions in OFDFT comes at the cost of approximating the electronic kinetic energy with a density functional (rather than the orbital-based expectation value of the Laplacian). State-of-the-art kinetic energy functionals include three contributions: ${ }^{9,10}$ the Thomas-Fermi contribution $T_{\mathrm{TF}}$, exact for a uniform electron gas, ${ }^{13,14}$ the von Weizsäcker contribution $T_{\mathrm{vW}}$, exact for a single orbital, ${ }^{15}$ and a densitydependent convolution term $T_{\text {ker }}$ that ensures the correct linear response of the electron gas (the Lindhard function). ${ }^{16}$ In the present simulation of Al, we use the Wang-Govind-Carter (WGC) KEDF. ${ }^{10,17}$ The WGC kernel is density dependent, which renders it more accurate than previous KEDFs. A second-order Taylor expansion of the kernel is employed to maintain $\mathcal{O}(N \ln N)$ scaling. ${ }^{10}$ Because the WGC KEDF follows the Lindhard linear response, it is most accurate for electrons in materials that are nearly-free-electron-like, such as the valence electrons in main group metals. Therefore, while in principle an all-electron version of OFDFT could be employed, instead we only solve explicitly for the density associated with the valence electrons. Consequently, a pseudopotential must be introduced to describe the effect of the core electrons plus nucleus on the valence electrons. In order to retain linear scaling, a local pseudopotential (LPS) that only depends on distance from the nucleus is used. Here we employ the Goodwin-Needs-Heine (GNH) empirical Al LPS. ${ }^{18}$ Some flexibility is lost when using spherically symmetric LPSs (rather than orbital-based nonlocal pseudopotentials), but the WGC-GNH combination yields vacancy formation energies and total energies for a variety of unstrained bulk phases that agree with KS-DFT to within 0.01 eV/atom. ${ }^{10}$

\section{Local calculations with OFDFT}

Local QC with OFDFT is used only in regions of the sample where the deformation gradient is smaller than that which would initiate nucleation of defects such as dislocations. As mentioned in Sec. II A, the energy of a finite element in the local region is computed using the Cauchy-Born rule, so that each finite element can be modeled by a single unit cell with periodic boundary conditions. The deformed geometry of each finite element is thus represented by a 3 $\times 3$ matrix $\mathbf{h}$, with the deformed primitive lattice vectors making up the columns of $\mathbf{h}$ [see Eq. (2)]. For each deformation gradient, an OFDFT energy/stress calculation is carried out by minimizing the electronic total energy.

The stress tensor in the OFDFT formulation is defined as follows:

$$
\sigma_{a b}=\frac{1}{\Omega} \sum_{\nu} \frac{\partial E}{\partial h_{a \nu}} h_{b \nu}
$$

with $\Omega$ as the volume of deformed unit cell and $h_{i j}$ denoting the component of the deformed lattice vector $h_{j}$ in Cartesian dimension $i$. 


\section{WGC kinetic energy and stress}

The WGC functional takes the form

$$
\begin{gathered}
T_{\mathrm{s}}[\rho]=T_{\mathrm{TF}}[\rho]+T_{\mathrm{vW}}[\rho]+T_{\mathrm{ker}}[\rho], \\
T_{\mathrm{TF}}=C_{\mathrm{TF}} \int \rho^{5 / 3}(\mathbf{r}) d \mathbf{r}, \\
T_{\mathrm{vW}}=\frac{1}{8} \int \frac{|\nabla \rho(\mathbf{r})|^{2}}{\rho(\mathbf{r})} d \mathbf{r}=-\frac{1}{2} \int \rho^{1 / 2}(\mathbf{r}) \nabla^{2} \rho^{1 / 2}(\mathbf{r}) d \mathbf{r}, \\
T_{\mathrm{ker}}=C_{\mathrm{TF}} \iint \rho^{\alpha}(\mathbf{r}) w_{\alpha \beta}\left[\xi_{\gamma}\left(\mathbf{r}, \mathbf{r}^{\prime}\right), \mathbf{r}-\mathbf{r}^{\prime}\right] \rho^{\beta}\left(\mathbf{r}^{\prime}\right) d \mathbf{r} d \mathbf{r}^{\prime},
\end{gathered}
$$

where $T_{\mathrm{TF}}, T_{\mathrm{vW}}$, and $T_{\mathrm{ker}}$ are the Thomas-Fermi functional, the von Weizsäcker functional, and kernel functionals, respectively, and $C_{\mathrm{TF}}=\frac{3}{10}\left(3 \pi^{2}\right)^{2 / 3}$. For $\mathrm{Al}$, the parameter values of $\{\alpha, \beta\}=\left\{\frac{5 \pm \sqrt{5}}{6}\right\}$ and $\gamma=2.7$. The variable

$$
\xi_{\gamma}\left(\mathbf{r}, \mathbf{r}^{\prime}\right)=\left[\frac{k_{F}^{\gamma}(\mathbf{r})+k_{F}^{\gamma}\left(\mathbf{r}^{\prime}\right)}{2}\right]^{1 / \gamma}
$$

is the two-body Fermi wave vector, and $k_{F}(\mathbf{r})=\left[3 \pi^{2} \rho(\mathbf{r})\right]^{1 / 3}$ is the local one-body Fermi wave vector.

As with the energy, the WGC stress is split up into a Thomas-Fermi, a von Weizsäcker, and a kernel term:

$$
\sigma_{a b}^{\mathrm{WGC}}=\sigma_{a b}^{\mathrm{TF}}+\sigma_{a b}^{\mathrm{vW}}+\sigma_{a b}^{\mathrm{ker}} .
$$

Thomas-Fermi stress has the form

$$
\sigma_{a b}^{\mathrm{TF}}=-\frac{2 T^{T F}}{3 \Omega} \delta_{a b}
$$

where $\delta_{a b}$ is the Kronecker delta. The von Weizsäcker stress can be written as

$$
\begin{aligned}
\sigma_{a b}^{\mathrm{vW}}= & -\frac{1}{4 \Omega} \int\left[\frac{\partial \rho(\mathbf{r})}{\partial r_{a}}\right]\left[\frac{\partial \rho(\mathbf{r})}{\partial r_{b}}\right] \frac{1}{\rho(\mathbf{r})} d \mathbf{r}= \\
& -\frac{1}{\Omega} \int \rho^{1 / 2}(\mathbf{r}) \frac{\partial^{2} \rho^{1 / 2}(\mathbf{r})}{\partial r_{a} \partial r_{b}} d \mathbf{r} .
\end{aligned}
$$

The stress derived from WGC density-dependent kernel has different values depending on whether average electron density $\rho_{*}$ or total number electrons $N_{e}$ remains constant as the cell's dimensions change. Here, we model an infinite bulk crystal in each finite element in the local QC regions, so $N_{e}$ is fixed to its undeformed value in each deformed unit cell; the stress is given by ${ }^{19}$

$$
\begin{aligned}
\sigma_{a b}^{\mathrm{ker}}= & \sum_{\mathbf{g} \neq 0}\left\{-\frac{1}{3} T_{\mathrm{WGC}}^{\alpha \beta \gamma} \delta_{a b}+C_{\mathrm{TF}} \eta_{* *}\left(\frac{g_{a} g_{b}}{|\mathbf{g}|^{2}}-\frac{1}{3} \delta_{a b}\right)\right. \\
& \times\left[\tilde{\rho}_{\alpha}(-\mathbf{g}) d_{00} \tilde{\rho}_{\beta}(\mathbf{g})+\tilde{\rho}_{\alpha+1}(-\mathbf{g}) d_{01} \tilde{\rho}_{\beta}(\mathbf{g})\right. \\
& +\tilde{\rho}_{\alpha}(-\mathbf{g}) d_{01} \tilde{\rho}_{\beta+1}(\mathbf{g})+\tilde{\boldsymbol{\rho}}_{\alpha+2}(-\mathbf{g}) d_{02} \tilde{\rho}_{\beta}(\mathbf{g}) \\
& \left.\left.+\tilde{\rho}_{\alpha}(-\mathbf{g}) d_{02} \tilde{\rho}_{\beta+2}(\mathbf{g})+\tilde{\rho}_{\alpha+1}(-\mathbf{g}) d_{11} \tilde{\rho}_{\beta+1}(\mathbf{g})\right]\right\},
\end{aligned}
$$

where $\eta_{*}=|\mathbf{g}| /\left(2 k_{F *}\right)$ is the dimensionless momentum assuming electron density $\rho_{*}, \mathbf{g}$ is a reciprocal space lattice vector,

$$
\begin{gathered}
\rho_{D}(\mathbf{g})=\mathcal{F}\left[\rho^{D}(\mathbf{r})\right], \\
d_{00}=\frac{\partial \mathcal{F}\left[w_{\alpha \beta}\right]}{\partial \eta_{*}}+2 \rho_{*} \frac{\partial \mathcal{F}\left[w_{\alpha \beta}^{\prime}\right]}{\partial \eta_{*}}-\rho_{*}^{2}\left(\frac{\partial \mathcal{F}\left[w_{\alpha \beta_{1}}^{\prime \prime}\right]}{\partial \eta_{*}}+\frac{\partial \mathcal{F}\left[w_{\alpha \beta_{2}}^{\prime \prime}\right]}{\partial \eta_{*}}\right), \\
d_{01}=-\frac{\partial \mathcal{F}\left[w_{\alpha \beta}^{\prime}\right]}{\partial \eta_{*}}+\rho_{*}\left(\frac{\partial \mathcal{F}\left[w_{\alpha \beta_{1}}^{\prime \prime}\right]}{\partial \eta_{*}}+\frac{\partial \mathcal{F}\left[w_{\alpha \beta_{2}}^{\prime \prime}\right]}{\partial \eta_{*}}\right) \\
d_{02}=-\frac{1}{2} \frac{\partial \mathcal{F}\left[w_{\alpha \beta_{1}}^{\prime \prime}\right]}{\partial \eta_{*}}, \\
d_{11}=-\frac{\partial \mathcal{F}\left[w_{\alpha \beta_{2}}^{\prime \prime}\right]}{\partial \eta_{*}},
\end{gathered}
$$

with $\mathcal{F}$ indicating a Fourier transform, coefficients $d_{00}, d_{01}, d_{02}$ evaluated at $\rho_{*}$, and derivatives of the kernel defined as

$$
\begin{aligned}
w_{\alpha \beta}^{\prime} & =\frac{\partial w_{\alpha \beta}\left[\zeta_{\gamma}\left(\mathbf{r}, \mathbf{r}^{\prime}\right),\left|\mathbf{r}-\mathbf{r}^{\prime}\right|\right]}{\partial \rho(\mathbf{r})}, \\
w_{\alpha \beta_{1}}^{\prime \prime} & =\frac{\partial^{2} w_{\alpha \beta}\left[\zeta_{\gamma}\left(\mathbf{r}, \mathbf{r}^{\prime}\right),\left|\mathbf{r}-\mathbf{r}^{\prime}\right|\right]}{\partial \rho^{2}(\mathbf{r})}, \\
w_{\alpha \beta_{2}}^{\prime \prime} & =\frac{\partial^{2} w_{\alpha \beta}\left[\zeta_{\gamma}\left(\mathbf{r}, \mathbf{r}^{\prime}\right),\left|\mathbf{r}-\mathbf{r}^{\prime}\right|\right]}{\partial \rho(\mathbf{r}) \partial \rho\left(\mathbf{r}^{\prime}\right)} .
\end{aligned}
$$

\section{Electron-ion energy and stress}

The electron-ion energy is summed in real space,

$$
\begin{gathered}
E_{\mathrm{e}-\mathrm{i}}=\int \rho(\mathbf{r}) V^{\mathrm{ion}}(\mathbf{r}) d \mathbf{r}, \\
V^{\mathrm{ion}}(\mathbf{r})=\frac{1}{\Omega} \overline{\mathcal{F}}\left[\sum_{I} \widetilde{V}_{I}^{\mathrm{psp}}(\mathbf{g}) e^{-i \mathbf{g} \cdot \mathbf{R}_{I}}\right],
\end{gathered}
$$

where $\mathbf{R}_{I}$ is the position of ion I, $\widetilde{V}_{I}^{\mathrm{psp}}$ the Fourier transform of the pseudopotential of ion I $\left(V_{I}^{\mathrm{psp}}\right)$, and $\overline{\mathcal{F}}$ an inverse Fourier transform.

The corresponding electron-ion stress is

$$
\sigma_{a b}^{\mathrm{e}-\mathrm{i}}=-\frac{1}{\Omega}\left[\sum_{\mathbf{g} \neq 0}\left(\tilde{\rho}(\mathbf{g}) \frac{g_{a} g_{b}}{|\mathbf{g}|} \sum_{I} \frac{\partial \widetilde{V}_{I}^{\mathrm{psp}}(|\mathbf{g}|)}{\partial|\mathbf{g}|} e^{i \mathbf{g} \cdot \mathbf{R}_{I}}\right)+\delta_{a b} E_{\mathrm{e}-\mathrm{i}}\right] .
$$

Note that this stress equation corrects Eq. (A3) in Ref. 20.

\section{Hartree energy and stress}

As in Ref. 20, the Hartree energy takes the form 


$$
E_{\mathrm{H}}=\frac{1}{2} \iint \frac{\rho(\mathbf{r}) \rho\left(\mathbf{r}^{\prime}\right)}{\left|\mathbf{r}-\mathbf{r}^{\prime}\right|} d \mathbf{r} d \mathbf{r}^{\prime}
$$

and the Hartree stress is

$$
\sigma_{a b}^{H}=\sum_{\mathbf{g} \neq 0} \frac{2 \pi}{|\mathbf{g}|^{2}}\left(\frac{2 g_{a} g_{b}}{|\mathbf{g}|^{2}}\right) \rho(\mathbf{g}) \rho(-\mathbf{g})-\delta_{a b} \frac{E_{\mathrm{H}}}{\Omega} .
$$

\section{Exchange-correlation energy and stress}

In this work, we use the local-density approximation $(\mathrm{LDA})^{21,22}$ for $E_{\mathrm{xc}}[\rho]$. As in Ref. 20 the LDA exchangecorrelation energy is expressed as

$$
E_{\mathrm{xc}}=\int \rho(\mathbf{r})\left\{\epsilon_{x}[\rho(\mathbf{r})]+\epsilon_{c}[\rho(\mathbf{r})]\right\} d \mathbf{r},
$$

where $\epsilon_{x}$ is the Slater-Dirac ${ }^{23}$ exchange energy density functional of a uniform electron gas of density $\rho$ and $\epsilon_{c}$ is the Perdew-Zunger ${ }^{21}$ parameterization of the Ceperley-Alder ${ }^{22}$ correlation energy density functional. The exchangecorrelation stress has the form

$$
\sigma_{a b}^{x c}=\frac{1}{\Omega}\left\{E_{\mathrm{xc}}-\int \rho(\mathbf{r})\left[\frac{\delta E_{\mathrm{xc}}}{\delta \rho(\mathbf{r})}\right] d \mathbf{r}\right\} \delta_{a b} .
$$

\section{Ewald energy and stress}

The Ewald summation calculates the ion-ion interactions for a periodic system. ${ }^{24,25}$ There is a short-range term in real space, a long-range term calculated in reciprocal space, a self-correction term, and a background correction. The background correction is necessary for systems that are not charge neutral, which is the case for our system, since the ionic interactions summed by the Ewald method are between cations stripped of their valence electrons. The ion-ion repulsion energy is expressed as

$$
\begin{aligned}
E^{\mathrm{i}-\mathrm{i}}= & \frac{1}{2} \sum_{\mathbf{L}} \sum_{I, J}^{\prime} Z_{I} Z_{J} \frac{\operatorname{erfc}\left(\eta\left|\mathbf{r}_{I J, \mathbf{L}}\right|\right)}{\left|\mathbf{r}_{I J, \mathbf{L}}\right|} \\
& +\frac{2 \pi}{\Omega} \sum_{\mathbf{g} \neq 0} \frac{\exp \left(-|\mathbf{g}|^{2} / 4 \eta^{2}\right)}{|\mathbf{g}|^{2}}\left\{\left[\sum_{I} Z_{I} \cos \left(\mathbf{g} \cdot \mathbf{R}_{I}\right)\right]^{2}\right. \\
& \left.+\left[\sum_{I} Z_{I} \sin \left(\mathbf{g} \cdot \mathbf{R}_{I}\right)\right]^{2}\right\}-\frac{\eta}{\sqrt{\pi}} \sum_{I} Z_{I}^{2}-\frac{\pi}{2 \Omega \eta^{2}}\left(\sum_{I} Z_{I}\right)^{2},
\end{aligned}
$$

where $I$ and $J$ indicate atoms within the periodic cell, $Z$ is the ionic charge, $R$ are the ionic positions, $\mathbf{L}$ indicates the origins of each image cell, and $\mathbf{r}_{\mathrm{IJ}, \mathrm{L}}=\mathbf{L}+\mathbf{R}_{I}-\mathbf{R}_{J}$. The prime over the summation indicates that we skip terms where both $I=J$ and $\mathbf{L}=0$. Note that here $\eta$ is a constant, unrelated to $\eta_{*}$ in the WGC stress functional. The error due to the Ewald energy is bounded to less than $10^{-10} \mathrm{eV}$ by on-the-fly calculation of the parameter $\eta$, the real space cutoff, and the reciprocal space cutoff. ${ }^{26}$ The Ewald stress is ${ }^{20}$

$$
\begin{aligned}
\sigma_{a b}^{\mathrm{i}-\mathrm{i}}= & -\frac{1}{2 \Omega} \sum_{\mathbf{L}} \sum_{I, J}^{\prime} Z_{I} Z_{J}\left\{\frac{2 \eta}{\sqrt{\pi}} \exp \left(-\eta^{2}\left|\mathbf{r}_{I J, L}\right|^{2}\right)\right. \\
& \left.+\frac{\operatorname{erfc}\left(\eta\left|\mathbf{r}_{I J, L}\right|\right)}{\left|\mathbf{r}_{I J, L}\right|}\right\} \frac{r_{I J, L, a} r_{I J, L, b}}{\left|r_{I J, L}\right|^{2}} \\
& +\frac{2 \pi}{\Omega^{2}} \sum_{\mathbf{g} \neq 0} \frac{\exp \left(-|\mathbf{g}|^{2} / 4 \eta^{2}\right)}{|\mathbf{g}|^{2}}\left\{\left[\sum_{I} Z_{I} \cos \left(\mathbf{g} \cdot \mathbf{R}_{I}\right)\right]^{2}\right. \\
& \left.+\left[\sum_{I} Z_{I} \sin \left(\mathbf{g} \cdot \mathbf{R}_{I}\right)\right]^{2}\right\} \\
& \times\left\{\frac{2 g_{a} g_{b}}{|\mathbf{g}|^{2}}\left(1+\frac{|\mathbf{g}|^{2}}{4 \eta^{2}}\right)-\delta_{a b}\right\}+\frac{\pi}{2 \Omega^{2} \eta^{2}}\left(\sum_{I} Z_{I}\right)^{2} \delta_{a b} .
\end{aligned}
$$

\section{Nonlocal calculations with OFDFT}

The nonlocal region is modeled at the atomistic level with a QM/MM approach. In a typical QM/MM calculation, the system is partitioned into two separated domains: a QM region and a MM region. In QCDFT, the QM region refers to the nonlocal region and the MM region refers to the buffer region. The buffer region is introduced to provide the boundary conditions for the calculation of nonlocal energy and it contains both dummy atoms and local repatoms. The dummy atoms differ from the local and nonlocal repatoms in the following sense: (i) their positions are interpolated from the positions of local repatoms using finite element shape functions; (ii) the energy and force on the dummy atoms do not need to be considered explicitly since they are not explicit degrees of the freedom in the $\mathrm{QC}$ formulation.

At present, there are two types of $\mathrm{QM} / \mathrm{MM}$ coupling strategies: mechanical coupling and quantum coupling. ${ }^{27-31}$ The interaction energy between the QM and MM regions is formulated at the MM level for mechanical coupling, and at the QM level for quantum coupling. The latter form should be more accurate as it accounts for all quantum mechanical interactions. Depending on the level of the quantum description, the extent of the electronic coupling varies from merely long-range electrostatic interaction to a full Coulomb interaction, including short-ranged exchange correlations. ${ }^{27,31}$ Here we use an OFDFT-based quantum mechanical QM/MM coupling proposed by Zhang and $\mathrm{Lu}^{5}$ which considers the full Coulomb, kinetic energy, and exchange-correlation interactions. More specifically, both the energy of the nonlocal atoms and the interaction energy between the nonlocal atoms and the buffer atoms are calculated by OFDFT. To simply the notation, we denote the nonlocal region as region I and the buffer region as region II. Typically, the buffer region consists of several atomic layers surrounding the nonlocal region. The buffer region has to be chosen carefully for a system of interest. Systematic study of the choice of the buffer region has been carried out previously for $\mathrm{Al}^{5}$ and we will use these results in the present paper (cf. Sec. III C for more details). The nonlocal energy $E^{\text {nl }}$ as defined in Eq. (4) can be expressed as

$$
E^{\mathrm{nl}}\left[\rho^{\mathrm{tot}}\right]=\min _{\rho}\left[E_{\mathrm{OF}}\left(\rho^{\mathrm{I}} ; \mathbf{R}^{\mathrm{I}}\right)+E_{\mathrm{OF}}^{\mathrm{int}}\left(\rho^{\mathrm{I}}, \rho^{\mathrm{II}} ; \mathbf{R}^{\mathrm{I}}, \mathbf{R}^{\mathrm{II}}\right)\right],
$$

where $\mathbf{R}^{\mathrm{I}}$ and $\mathbf{R}^{\mathrm{II}}$ denote ionic coordinates in region I and II, respectively. The OFDFT energy functional $E_{\mathrm{OF}}$ has been 


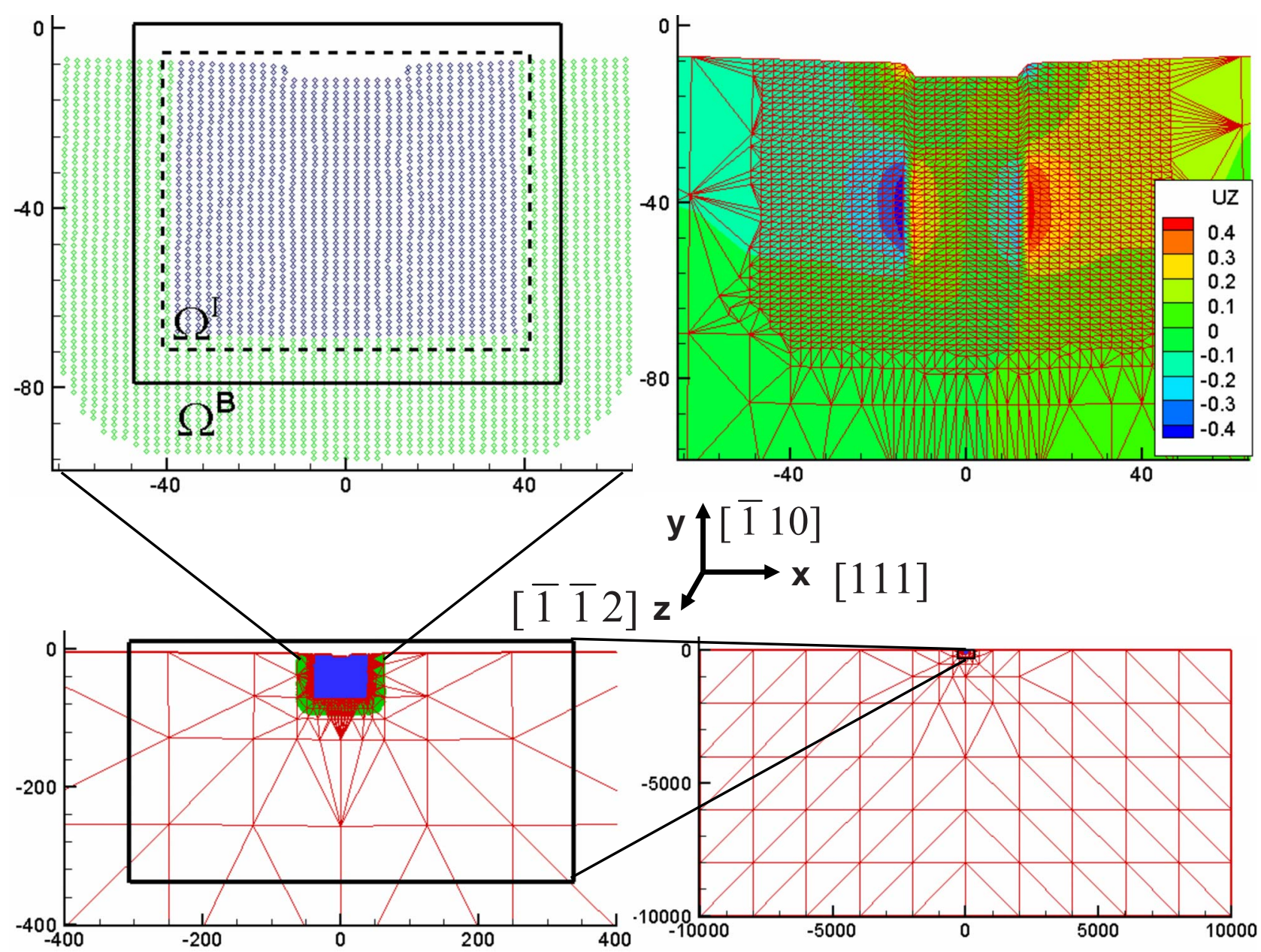

FIG. 1. (Color online) The overview of the entire system and domain partition in QCDFT with nanoindentation as an example. The $x, y$

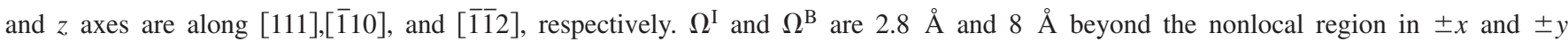
directions, respectively (Ref. 5). The colors indicate $u_{z}$, the out-of-plane displacement of atoms in the $z$ direction.

introduced earlier [Eq. (5)]. The total charge density of the QM/MM system $\rho^{\text {tot }}$ consists of two contributions: $\rho^{\text {tot }}=\rho^{\mathrm{I}}$ $+\rho^{\mathrm{II}}$, where $\rho^{\mathrm{I}}$ and $\rho^{\mathrm{II}}$ represent the charge density from region $\mathrm{I}$ and II, respectively. While $\rho^{\mathrm{I}}$ is determined selfconsistently by minimizing the total energy functional Eq. (33), $\rho^{\mathrm{II}}$ is defined as a superposition of atom-centered charge densities $\rho^{\text {at }}$ via $\rho^{\mathrm{II}}(\mathbf{r})=\sum_{i \in \mathrm{II}} \rho^{\text {at }}\left(\mathbf{r}-\mathbf{R}_{i}\right)$. Note that $\rho^{\text {at }}$ is spherically symmetric and can be constructed a priori. It is important to point out that $\rho^{\text {at }}$ is not a charge density of an isolated atom, but rather an atom-centered charge density whose superposition gives rise to the approximate bulk density of region II. ${ }^{5}$ It should be noted that in general, the atom-centered charge density should contain higher angular momentum contributions, including, e.g., $p$ - and $d$-like densities. For aluminum, which is the system of interest in the present paper, we found that the $s$-like charge density alone could give sufficiently good results. ${ }^{5}$ Therefore $\rho^{\mathrm{II}}(\mathbf{r})$ is fixed for a given ionic configuration of region II and it changes upon the motion of region II ions. In other words, the electronic degree of freedom in the formulation is $\rho^{\mathrm{I}}$ only and $\rho^{\mathrm{II}}$ is fixed during the electronic relaxation. The interaction energy is thus defined as follows:

$$
\begin{aligned}
E_{\mathrm{OF}}^{\mathrm{int}}\left[\rho^{\mathrm{I}}, \rho^{\mathrm{II}} ; \mathbf{R}^{\mathrm{I}}, \mathbf{R}^{\mathrm{II}}\right]= & E_{\mathrm{OF}}\left[\rho^{\mathrm{tot}} ; \mathbf{R}^{\mathrm{tot}}\right]-E_{\mathrm{OF}}\left[\rho^{\mathrm{I}} ; \mathbf{R}^{\mathrm{I}}\right] \\
& -E_{\mathrm{OF}}\left[\rho^{\mathrm{II}} ; \mathbf{R}^{\mathrm{II}}\right],
\end{aligned}
$$

where $\mathbf{R}^{\text {tot }} \equiv \mathbf{R}^{\mathrm{I}} \cup \mathbf{R}^{\mathrm{II}}$. The energy functional of Eq. (33) can be written as

$$
E^{\mathrm{nl}}\left[\rho^{\mathrm{tot}}\right]=\min _{\rho^{\mathrm{I}}}\left[E_{\mathrm{OF}}\left(\rho^{\mathrm{tot}} ; \mathbf{R}^{\mathrm{tot}}\right)-E_{\mathrm{OF}}\left(\rho^{\mathrm{II}} ; \mathbf{R}^{\mathrm{II}}\right)\right] .
$$

A basic ansatz of the present $\mathrm{QM} / \mathrm{MM}$ formulation [Eq (35)] is that $\rho^{\mathrm{I}}$ must be confined within a finite volume $\left(\Omega^{\mathrm{I}}\right)$ that is necessarily larger than region I but much smaller than the entire QM/MM region. The confinement of $\rho^{\mathrm{I}}$ is enforced by setting $\rho^{\mathrm{I}}$ to be zero outside $\Omega^{\mathrm{I}}$. Therefore beyond $\Omega^{\mathrm{I}}, \rho^{\mathrm{II}}$ should reproduce the correct total density, $\rho^{\text {tot }}$. In addition, since some terms in the formulation [Eq. (35)] could be more efficiently computed in reciprocal space (discussed in the following), we also introduce a volume $\Omega^{\mathrm{B}}$ over which the periodic boundary conditions are applied. The periodic box $\Omega^{\mathrm{B}}$ should be larger than $\Omega^{\mathrm{I}}$ so that $\rho^{\mathrm{I}}$ does not overlap with its periodic images. ${ }^{5}$ Note that the $\mathrm{QM} / \mathrm{MM}$ system is only a small fraction of the entire QCDFT system. To facilitate the introduction of the QCDFT method, we present a schematic 
diagram in Fig. 1 which demonstrates the typical partition of domains in a QCDFT calculation. This particular example is for a nanoindentation calculation of an $\mathrm{Al}$ thin film which is used to validate the QCDFT method (see Sec. III for details). The lower-right panel shows the entire system and the corresponding finite element mesh. The lower-left panel is a blow-up view of the entire system, which is further zoomed in as shown in the upper-left panel, focusing on the nonlocal region. The upper-right panel shows out-of-plane displacements of the nonlocal atoms, where the dislocations and the stacking faults are clearly visible. All lengths are given in $\AA$. The blue and green circles represent the nonlocal and buffer atoms, respectively. The volumes $\Omega^{\mathrm{I}}$ and $\Omega^{\mathrm{B}}$ are represented by the black dashed box and solid box in the upper-left panel, respectively. There is no constraint on $\rho^{\mathrm{II}}$, which can extend to the entire QM/MM system. In addition to its computational efficiency as discussed in Sec. II B, OFDFT allows Eq. (35) to be evaluated over $\Omega^{\mathrm{I}}$ rather than over the entire QM/MM system as Eq. (35) appears to suggest. ${ }^{5,32}$ This significant computational saving is due to the cancellation in evaluating the first and second term of Eq. (35), and it is rendered by the orbital-free nature of OFDFT and the localization of $\rho^{\mathrm{I}}$. In the following, we will express all terms in Eq. (35), and the cancellation can be seen explicitly from these expressions. It should be noted that as the deformation evolves, the number of nonlocal repatoms and the valence electrons could change from one nonlocal OFDFT calculation to the next, however, the number of the valence electrons in each nonlocal OFDFT calculation remains constant for a given set of nonlocal repatoms.

\section{Noninteracting kinetic energy terms}

First we consider the local terms of the kinetic energy functional, $T_{\mathrm{TF}}$ and $T_{\mathrm{vW}}$, which are given by

$$
\begin{aligned}
T_{\mathrm{TF}}\left[\rho^{\mathrm{tot}}\right]-T_{\mathrm{TF}}\left[\rho^{\mathrm{II}}\right] & =\int f_{\mathrm{TF}}\left(\rho^{\mathrm{tot}}\right) d \mathbf{r}-\int f_{\mathrm{TF}}\left(\rho^{\mathrm{II}}\right) d \mathbf{r} \\
& =\int_{\Omega^{\mathrm{I}}}\left[f_{\mathrm{TF}}\left(\rho^{\mathrm{tot}}\right)-f_{\mathrm{TF}}\left(\rho^{\mathrm{II}}\right)\right] d \mathbf{r}, \\
T_{\mathrm{vW}}\left[\rho^{\mathrm{tot}}\right]-T_{\mathrm{vW}}\left[\rho^{\mathrm{II}}\right] & =\int f_{\mathrm{vW}}\left(\rho^{\mathrm{tot}}\right) d \mathbf{r}-\int f_{\mathrm{vW}}\left(\rho^{\mathrm{II}}\right) d \mathbf{r} \\
& =\int_{\Omega^{\mathrm{I}}}\left[f_{\mathrm{vW}}\left(\rho^{\mathrm{tot}}\right)-f_{\mathrm{vW}}\left(\rho^{\mathrm{II}}\right)\right] d \mathbf{r},
\end{aligned}
$$

where $f_{\mathrm{TF}}(\rho)=\frac{3}{10}\left(3 \pi^{2}\right)^{2 / 3} \rho^{5 / 3}, f_{\mathrm{vW}}(\rho)=-\frac{1}{2} \rho^{1 / 2} \nabla^{2} \rho^{1 / 2}$ and we have used the fact that $\rho^{\text {tot }}(\mathbf{r})=\rho^{\mathrm{II}}(\mathbf{r})$ when $\mathbf{r}$ is beyond $\Omega^{\mathrm{I}}$. Therefore the calculations involve an integral over $\Omega^{\mathrm{I}}$ rather than over the entire system. It is clear that the TF term can be computed easily and efficiently on a grid in real space within $\Omega^{\mathrm{I}}$. Finite differencing schemes allow the $\mathrm{vW}$ term to be evaluated on only $\Omega^{\mathrm{I}}$ as well. However, in practice, the vW term is computed in reciprocal space which is more efficient. This allows greater accuracy, but as a tradeoff, its evaluation requires integration over $\Omega^{\mathrm{B}}$.

The kernel term of the kinetic energy has a complicated nonlocal form which requires close scrutiny:

$$
\begin{aligned}
T_{\mathrm{ker}}\left[\rho^{\mathrm{tot}}\right]-T_{\mathrm{ker}}\left[\rho^{\mathrm{II}}\right]= & \int_{\Omega^{\mathrm{I}}} F(\mathbf{r})(K * G)(\mathbf{r}) d \mathbf{r} \\
& +\int_{\Omega^{\mathrm{I}}} F(\mathbf{r})\left(K * g_{2}\right)(\mathbf{r}) d \mathbf{r} \\
& +\int_{\Omega^{\mathrm{I}}} G(\mathbf{r})\left(K * f_{2}\right)(\mathbf{r}) d \mathbf{r},
\end{aligned}
$$

where the convolution integral is defined as $(K * G)(\mathbf{r})$ $\equiv \int K\left(\mathbf{r}-\mathbf{r}^{\prime}\right) G\left(\mathbf{r}^{\prime}\right) d \mathbf{r}^{\prime}$, etc. and

$$
\begin{aligned}
& f_{2}(\mathbf{r}) \equiv f\left(\rho^{\mathrm{II}}(\mathbf{r})\right), \quad f_{12}(\mathbf{r}) \equiv f\left(\rho^{\mathrm{I}}(\mathbf{r})+\rho^{\mathrm{II}}(\mathbf{r})\right), \\
& g_{2}(\mathbf{r}) \equiv g\left(\rho^{\mathrm{II}}(\mathbf{r})\right), \quad g_{12}(\mathbf{r}) \equiv g\left(\rho^{\mathrm{I}}(\mathbf{r})+\rho^{\mathrm{II}}(\mathbf{r})\right), \\
& F(\mathbf{r}) \equiv f_{12}(\mathbf{r})-f_{2}(\mathbf{r}), \quad G(\mathbf{r}) \equiv g_{12}(\mathbf{r})-g_{2}(\mathbf{r}) .
\end{aligned}
$$

With the WGC $\mathrm{KEDF}^{10} f(x)=x^{\alpha}, \quad g(x)=x^{\beta}$, and $K$ $=C_{\mathrm{TF}} w_{\alpha \beta}\left[\xi_{\gamma}\left(\mathbf{r}, \mathbf{r}^{\prime}\right), \mathbf{r}-\mathbf{r}^{\prime}\right]$, as in Eq. (10). While the kernel term $T_{\text {ker }}\left[\rho^{\text {tot }}\right]-T_{\text {ker }}\left[\rho^{\mathrm{II}}\right]$ is expressed as integrals over $\Omega^{\mathrm{I}}$, the time-consuming convolution terms, such as $(K * G)(\mathbf{r})$ are actually integrated over the entire space. We have shown that the fast Fourier transforms (FFT) can be used to make such computations both efficient and accurate, ${ }^{5}$ where the evaluation of convolution terms are confined within $\Omega^{\mathrm{B}}$.

\section{Electrostatic Coulomb energy terms}

According to Eq. (33), we can express the electrostatic terms as $\left(E_{\mathrm{H}}\left[\rho^{\mathrm{I}}\right]+E_{\mathrm{e}-\mathrm{i}}\left[\rho^{\mathrm{I}}\right]+E_{\mathrm{i}-\mathrm{i}}\left(\mathbf{R}^{\mathrm{I}}\right)\right)+\left(E_{\mathrm{H}}^{\mathrm{int}}+E_{\mathrm{e}-\mathrm{i}}^{\mathrm{int}}+E_{\mathrm{i}-\mathrm{i}}^{\mathrm{int}}\right)$, where the first three terms represent the electrostatic energy of region I, and the last three terms represent the electrostatic contribution of the interaction energy. The electrostatic energy of region I can be evaluated within $\Omega^{\mathrm{I}}$ :

$$
\begin{aligned}
\left(E_{\mathrm{H}}\left[\rho^{\mathrm{I}}\right]+E_{\mathrm{e}-\mathrm{i}}\left[\rho^{\mathrm{I}}\right]+E_{\mathrm{i}-\mathrm{i}}\left(\mathbf{R}^{\mathrm{I}}\right)\right) \\
=\frac{1}{2} \int_{\Omega^{\mathrm{I}}} \frac{\rho^{\mathrm{I}}(\mathbf{r}) \rho^{\mathrm{I}}\left(\mathbf{r}^{\prime}\right)}{\left|\mathbf{r}-\mathbf{r}^{\prime}\right|} d \mathbf{r} d \mathbf{r}^{\prime} \\
\quad+\sum_{i} \int_{\Omega^{\mathrm{I}}} \rho^{\mathrm{I}}(\mathbf{r}) V_{\mathrm{psp}}\left(\mathbf{r}-\mathbf{R}_{\mathrm{i}}^{\mathrm{I}}\right) d \mathbf{r}+\sum_{i<j}^{\mathrm{I}} \frac{Z_{i} Z_{j}}{\left|\mathbf{R}_{i}-\mathbf{R}_{j}\right|} .
\end{aligned}
$$

For the electrostatic contribution of the interaction energy, the long-range $1 / R$ Coulomb interactions in electronelectron, electron-ion and ion-ion contributions are canceled identically for a charge-neutral system. Thus it is convenient to group the three terms together when evaluating the electrostatic energies. The sum of the three terms can be written as

$$
\begin{aligned}
\left(E_{\mathrm{H}}^{\mathrm{int}}\right. & \left.+E_{\mathrm{e}-\mathrm{i}}^{\mathrm{int}}+E_{\mathrm{i}-\mathrm{i}}^{\mathrm{int}}\right) \\
& =\int_{\Omega^{\mathrm{I}}} \rho^{\mathrm{I}}(\mathbf{r})\left[\sum_{i} V_{\mathrm{elec}}^{\mathrm{at}}\left(\mathbf{r}-\mathbf{R}_{i}^{\mathrm{II}}\right)\right] d \mathbf{r}+\sum_{i, j} \chi\left(\mathbf{R}_{i}^{\mathrm{I}}-\mathbf{R}_{j}^{\mathrm{II}}\right),
\end{aligned}
$$

where we define 


$$
\begin{gathered}
V_{\mathrm{elec}}^{\mathrm{at}}(\mathbf{r}) \equiv \int \frac{\rho^{\mathrm{at}}\left(\mathbf{r}^{\prime}\right)}{\left|\mathbf{r}-\mathbf{r}^{\prime}\right|} d \mathbf{r}^{\prime}+V_{\mathrm{psp}}(\mathbf{r}), \\
\chi\left(\mathbf{R}_{i}^{\mathrm{I}}-\mathbf{R}_{j}^{\mathrm{II}}\right) \equiv \int V_{\mathrm{psp}}\left(\mathbf{r}-\mathbf{R}_{i}^{\mathrm{I}}\right) \rho^{\mathrm{at}}\left(\mathbf{r}-\mathbf{R}_{j}^{\mathrm{II}}\right) d \mathbf{r}+\frac{Z_{i} Z_{j}}{\left|\mathbf{R}_{i}^{\mathrm{I}}-\mathbf{R}_{j}^{\mathrm{II}}\right|} .
\end{gathered}
$$

$V_{\text {elec }}^{\text {at }}\left(\mathbf{r}-\mathbf{R}_{i}^{\mathrm{II}}\right)$ represents the Coulomb potential at a point $\mathbf{r}$ in $\Omega^{1}$ due to the $i$ th atom in region II, and $\chi\left(\mathbf{R}_{i}^{\mathrm{I}}-\mathbf{R}_{j}^{\mathrm{II}}\right)$ represents the electrostatic Coulomb energy between the $i$ th ion in region I and the $j$ th atom in region II. Here we define an atom as an entity composed of an ion and its valence electrons. Due to the spherical symmetry of $\rho^{\text {at }}$ and $V_{\mathrm{psp}}, V_{\text {elec }}^{\text {at }}$ and $\chi$ are the functions of distance only. Both $V_{\text {elec }}^{\text {at }}(\mathbf{r})$ and $\chi(\mathbf{R})$ in Eq. (40) are short-ranged functions where the long-range $1 / R$ contributions have been canceled. Therefore the calculation of Eq. (40) is also confined to a smaller volume than the entire QM/MM system, determined by a cut-off distance beyond which the $1 / R$ relation is exact. The actual calculation of the electrostatic terms is performed in real space.

\section{Exchange-correlation energy terms}

Similar to $T_{\mathrm{TF}}$ and $T_{\mathrm{vW}}$ terms, the exchange-correlation energy is also a local term which is given by

$$
\begin{aligned}
E_{\mathrm{xc}}\left[\rho^{\mathrm{tot}}\right]-E_{\mathrm{xc}}\left[\rho^{\mathrm{II}}\right] & =\int f_{\mathrm{xc}}\left(\rho^{\mathrm{tot}}\right) d \mathbf{r}-\int f_{\mathrm{xc}}\left(\rho^{\mathrm{II}}\right) d \mathbf{r} \\
& =\int_{\Omega^{\mathrm{I}}}\left[f_{\mathrm{xc}}\left(\rho^{\mathrm{tot}}\right)-f_{\mathrm{xc}}\left(\rho^{\mathrm{II}}\right)\right] d \mathbf{r},
\end{aligned}
$$

where $f_{\mathrm{xc}}(\rho) \equiv \rho \varepsilon_{\mathrm{xc}}(\rho)$, and it can be evaluated efficiently in real space.

\section{Calculation of ionic force}

The ionic force is calculated by varying the total energy functional [Eq. (33)] with respect to the ionic displacement. At the ground state of the electrons, $\rho_{\min }^{\mathrm{I}}$ satisfies $\delta\left\{E_{\mathrm{OF}}[\mathrm{I}]\right.$ $\left.+E_{\mathrm{OF}}^{\mathrm{int}}\right\} /\left.\delta \rho^{\mathrm{I}}\right|_{\rho_{\min }^{\mathrm{I}}}=0$, therefore the ionic force in region $\mathrm{I}\left(\mathbf{F}_{i \in \mathrm{I}}\right)$ can be written as

$$
\begin{aligned}
-\mathbf{F}_{i \in \mathrm{I}}= & \frac{\partial\left\{E_{\mathrm{OF}}[\mathrm{I}]+E_{\mathrm{OF}}^{\mathrm{int}}\right\}}{\partial \mathbf{R}_{i}} \\
= & \int_{\Omega^{\mathrm{I}}} \rho^{\mathrm{I}}(\mathbf{r}) \nabla_{\mathbf{R}_{i}} V_{\mathrm{psp}}\left(\mathbf{r}-\mathbf{R}_{i}\right) d \mathbf{r} \\
& +\sum_{j \in \mathrm{II}} \nabla_{\mathbf{R}_{i}} \chi\left(\mathbf{R}_{i}-\mathbf{R}_{j}\right) .
\end{aligned}
$$

Since the second term in Eq. (43) is short-ranged, only the region II ions which are adjacent to the QM/MM interface contribute to the sum. The force contribution on region II ions due to the interaction energy turns out to be much more complicated because the interaction energy is not minimized with respect to $\rho^{\mathrm{II}}$, and thus, there are force contributions from the variation of $\rho^{\mathrm{II}}$. The force on the $j$ th ion in region II due to the interaction energy is given by

$$
-\mathbf{F}_{j \in \mathrm{II}}^{\mathrm{int}}=\frac{\partial E_{\mathrm{TF}+\mathrm{vW}+\mathrm{ker}}^{\mathrm{int}}}{\partial \mathbf{R}_{j}}+\frac{\partial\left[E_{\mathrm{H}}^{\mathrm{int}}+E_{\mathrm{e}-\mathrm{i}}^{\mathrm{int}}+E_{\mathrm{i}-\mathrm{i}}^{\mathrm{int}}\right]}{\partial \mathbf{R}_{j}}+\frac{\partial E_{\mathrm{xc}}^{\mathrm{int}}}{\partial \mathbf{R}_{j}},
$$

where the various contributions of the first term are given as

$$
\begin{gathered}
\frac{\partial T_{\mathrm{TF}}^{\mathrm{int}}}{\partial \mathbf{R}_{j}}=\int_{\Omega^{\mathrm{I}}} \nabla_{\mathbf{R}_{j}} \rho^{\mathrm{at}}\left(\mathbf{r}-\mathbf{R}_{j}\right)\left[\mu_{\mathrm{TF}}\left(\rho^{\mathrm{I}}+\rho^{\mathrm{II}}\right)-\mu_{\mathrm{TF}}\left(\rho^{\mathrm{II}}\right)\right] d \mathbf{r}, \\
\frac{\partial T_{\mathrm{vW}}^{\mathrm{int}}}{\partial \mathbf{R}_{j}}=\int_{\Omega^{\mathrm{I}}} \nabla_{\mathbf{R}_{j}} \rho^{\mathrm{at}}\left(\mathbf{r}-\mathbf{R}_{j}\right)\left[\mu_{\mathrm{vW}}\left(\rho^{\mathrm{I}}+\rho^{\mathrm{II}}\right)-\mu_{\mathrm{vW}}\left(\rho^{\mathrm{II}}\right)\right] d \mathbf{r}, \\
\frac{\partial T_{\mathrm{ker}}^{\mathrm{int}}}{\partial \mathbf{R}_{j}}=\int_{\Omega^{\mathrm{I}}} \nabla_{\mathbf{R}_{j}} \rho^{\mathrm{at}}\left(\mathbf{r}-\mathbf{R}_{j}\right)\left[f_{12}^{\prime}(\mathbf{r})(K * G)(\mathbf{r})+g_{12}^{\prime}(\mathbf{r})(K * F)\right. \\
\left.\quad \times(\mathbf{r})+F^{\prime}(\mathbf{r})\left(K * g_{2}\right)(\mathbf{r})+G^{\prime}(\mathbf{r})\left(K * f_{2}\right)(\mathbf{r})\right] d \mathbf{r} .(45
\end{gathered}
$$

The second term is given by

$$
\begin{aligned}
\frac{\partial\left[E_{\mathrm{H}}^{\mathrm{int}}+E_{\mathrm{e}-\mathrm{i}}^{\mathrm{int}}+E_{\mathrm{i}-\mathrm{i}}^{\mathrm{int}}\right]}{\partial \mathbf{R}_{j}}= & \sum_{i \in \mathrm{I}} \nabla_{\mathbf{R}_{j}} \chi\left(\mathbf{R}_{i}-\mathbf{R}_{j}\right) \\
& +\int_{\Omega^{\mathrm{I}}} \rho^{\mathrm{I}}(\mathbf{r}) \nabla_{\mathbf{R}_{j}} V_{\text {elec }}^{\text {at }}\left(\mathbf{r}-\mathbf{R}_{j}\right) d \mathbf{r},
\end{aligned}
$$

and the third term is given by

$$
\frac{\partial\left[E_{\mathrm{xc}}^{\mathrm{int}}\right]}{\partial \mathbf{R}_{j}}=\int_{\Omega^{\mathrm{I}}} \nabla_{\mathbf{R}_{j}} \rho^{\mathrm{at}}\left(\mathbf{r}-\mathbf{R}_{j}\right)\left[\mu_{\mathrm{xc}}\left(\rho^{\mathrm{I}}+\rho^{\mathrm{II}}\right)-\mu_{\mathrm{xc}}\left(\rho^{\mathrm{II}}\right)\right] d \mathbf{r} .
$$

In Eqs. (45)-(47), various $\mu$ terms are the derivative of corresponding energy functional with respect to $\rho^{\text {at }}(\mathbf{r})$. All the integrations are performed within $\Omega^{\mathrm{I}}$, and $\mathbf{F}_{j \in \mathrm{II}}^{\mathrm{int}}$ is nonzero only for the region II ions which are adjacent to the interface.

\section{E. Ghost forces}

The QC partitioning of the physical system into two regions causes an unphysical mismatch between the energy functional evaluated in the local and nonlocal regions. As a result, a seemingly well-defined energy functional for the entire QC model will lead to spurious forces near the interface called "ghost forces" in the QC literature. Note that the ghost force only exists on local repatoms adjacent to the local/nonlocal (or QM/MM) interface. The principal reason for the ghost force is that we choose to focus on approximating the energy and not the force. One could opt to avoid the ghost force by formulating the force appropriately, but then one could no longer define an appropriate total energy of the system. There are two advantages of having a well-defined energy in atomistic simulations: (i) it is numerically more efficient to minimize energy, compared to the absolute value of a force; (ii) one can potentially obey an energy conservation law in dynamical simulations.

In the QCDFT (or the QM/MM) approach, the ghost force is defined as the force difference between two distinctive 
formulations: (i) where the force is calculated by applying the Cauchy-Born rule throughout the entire system; this corresponds to a "consistent" way of calculating force, thus no ghost force exists and (ii) where the force is calculated based on the mixed local/nonlocal formulation aforementioned and hence the ghost force exists. In the first case, the force on a local repatom would be

$$
\tilde{\mathbf{F}}\left(\mathbf{R}^{\mathrm{II}}\right)=-\frac{\partial E_{\mathrm{CB}}\left(\mathbf{R}^{\mathrm{tot}}\right)}{\partial \mathbf{R}^{\mathrm{II}}},
$$

where $E_{\mathrm{CB}}\left(\mathbf{R}^{\mathrm{tot}}\right)$ is the total energy of the system where the Cauchy-Born rule is used throughout. In the second case, the total energy of the QM/MM system can be written as

$$
E^{\mathrm{tot}}\left(\mathbf{R}^{\mathrm{tot}}\right)=E_{\mathrm{CB}}\left(\mathbf{R}^{\mathrm{II}}\right)+E_{\mathrm{QM}}\left(\mathbf{R}^{\mathrm{I}}\right)+E_{\mathrm{QM}}^{\mathrm{int}}\left(\mathbf{R}^{\mathrm{tot}}\right),
$$

where $E_{\mathrm{CB}}\left(\mathbf{R}^{\mathrm{II}}\right)$ is the local energy computed using the Cauchy-Born rule, $E_{\mathrm{QM}}\left(\mathbf{R}^{\mathrm{I}}\right)$ is the nonlocal energy computed by a quantum mechanical approach, and $E_{\mathrm{QM}}^{\mathrm{int}}\left(\mathbf{R}^{\text {tot }}\right)$ is the quantum mechanical interaction energy. The force derived from this total energy functional is

$$
\begin{aligned}
\mathbf{F}\left(\mathbf{R}^{\mathrm{II}}\right) & =-\frac{\partial E_{\mathrm{CB}}\left(\mathbf{R}^{\mathrm{II}}\right)}{\partial \mathbf{R}^{\mathrm{II}}}-\frac{\partial E_{\mathrm{QM}}^{\mathrm{int}}\left(\mathbf{R}^{\mathrm{tot}}\right)}{\partial \mathbf{R}^{\mathrm{II}}} \\
& =-\frac{\partial E_{\mathrm{CB}}\left(\mathbf{R}^{\mathrm{tot}}\right)}{\partial \mathbf{R}^{\mathrm{II}}}+\frac{\partial E_{\mathrm{CB}}^{\mathrm{int}}\left(\mathbf{R}^{\mathrm{tot}}\right)}{\partial \mathbf{R}^{\mathrm{II}}}-\frac{\partial E_{\mathrm{QM}}^{\mathrm{int}}\left(\mathbf{R}^{\mathrm{tot}}\right)}{\partial \mathbf{R}^{\mathrm{II}}} \\
& =\widetilde{\mathbf{F}}\left(\mathbf{R}^{\mathrm{II}}\right)+\mathbf{F}^{\text {ghost }},
\end{aligned}
$$

where $E_{\mathrm{CB}}^{\text {int }}\left(\mathbf{R}^{\text {tot }}\right)=E_{\mathrm{CB}}\left(\mathbf{R}^{\text {tot }}\right)-E_{\mathrm{CB}}\left(\mathbf{R}^{\mathrm{I}}\right)-E_{\mathrm{CB}}\left(\mathbf{R}^{\mathrm{II}}\right)$ is the interaction energy calculated with the Cauchy-Born rule applied to the entire system, and $\mathbf{F}^{\text {ghost }}=\frac{\partial\left\{E_{\mathrm{CB}}^{\mathrm{int}}\left(\mathbf{R}^{\mathrm{tot})}\right)-E_{\mathrm{QM}}^{\mathrm{int}}\left(\mathbf{R}^{\mathrm{tot}}\right)\right\}}{\partial \mathbf{R}^{\mathrm{II}}}$ is the ghost force. Note that $E_{\mathrm{CB}}\left(\mathbf{R}^{\mathrm{I}}\right)$ does not depend on $\mathbf{R}^{\mathrm{II}}$, hence its contribution to force is zero.

Having determined the ghost force in QCDFT, one can correct for it by adding a correction force $\mathbf{F}^{\text {corr }}=-\mathbf{F}^{\text {ghost }}$ on the local atoms $\left(\mathbf{F}^{\text {corr }}+\mathbf{F}\left(\mathbf{R}^{\mathrm{II}}\right)\right)$ so that the resultant force is $\tilde{\mathbf{F}}\left(\mathbf{R}^{\mathrm{II}}\right)$. The correction force is applied to the local repatoms as a dead load, computed each time the status of the representative atoms is updated, and remains fixed until the next update required due to the evolving state of deformation. In practice, the correction force is nonzero only for the local repatoms adjacent to the nonlocal region. Finally, the total energy expression has to be modified accordingly so that its gradient is consistent with the new formulation of force. This is achieved by incorporating the work done by the correction force into the original energy formulation:

$$
\widetilde{E}^{\text {tot }}=E^{\text {tot }}-\sum_{\alpha}^{N_{\text {rep }}^{\prime}} \mathbf{F}_{\alpha}^{\mathrm{corr}} \cdot \mathbf{u}_{\alpha},
$$

where $N_{\text {rep }}^{\prime}$ denotes the number of local repatoms whose correction force is nonzero, and $\mathbf{u}_{\alpha}$ is the displacement of the $\alpha$ th local repatom.

\section{F. Parallelization of QCDFT}

The present QCDFT code is parallelized based on the Message Passing Interface (MPI). The parallelization is achieved for both local and nonlocal calculations. The parallelization for the local part is trivial: Since the energy/force calculation for each finite element is independent from others, one can divide the local calculations evenly onto each processor. The computational time is thus proportional to the ratio of the number of the local finite elements to the available processors. Parallelization of the nonlocal region is achieved through domain decomposition, since the calculations (e.g., charge density, energy and force computations) are all performed on real-space grids, except the convolution terms in the kinetic energy. Grid points are evenly distributed to available processors, and results are obtained by summing up contributions from all grids. The calculation of the convolution terms is performed by parallelized FFT.

\section{G. Comparisons to earlier QCDFT schemes}

Efforts to combine QC with quantum simulations began as soon as the original QC method was introduced. Early work involved using quantum mechanical methods to compute the strain energy density and stress in a local QC (LQC) formulation. For example, Smith et al. ${ }^{33,34}$ incorporated a DFT-based tight-binding model into the LQC formulation for a nanoindentation study of Si. Tadmor et ll $^{35}$ used the LQC framework with DFT-based effective Hamiltonians to study polarization switching in $\mathrm{PbTiO}_{3}$. More recently, Hayes et al. ${ }^{19,20}$ implemented OFDFT in LQC to study nanoindentation of $\mathrm{Al}$ and $\mathrm{Al}_{3} \mathrm{Mg}$. These methods were valuable for providing physical insights that would be otherwise unattainable by empirical atomistic simulations, but being based only on LQC, they could not treat lattice defects such as dislocations, cracks, etc. explicitly. Lu et al. ${ }^{36}$ introduced the first QCDFT method which included the full QC method-mixing local and nonlocal formulation-with KS-DFT. In this version of QCDFT, the nonlocal region was treated by an embedded atom method (EAM)-based QM/MM coupling approach: KS-DFT was coupled to EAM with the interaction energy calculated classically by EAM. The local region, on the other hand, was dealt with by EAM. This method has been used to study dislocation ${ }^{36}$ and crack $^{37}$ properties in the presence of hydrogen impurities in Al. The major advantage of the method is its simplicity and versatility. Compared to the present QCDFT method, the downsides of the original QCDFT method are the following: (i) QM/MM coupling in the nonlocal region was not treated as accurately and rigorously as the present method; (ii) two different energy functionals are used in the formulation, KS-DFT and EAM; thus additional errors could arise due to this discrepancy. Recently, the so-called QC-OFDFT method $^{38}$ was proposed based on the coarse-graining idea of QC with OFDFT as the sole energy formulation of the method. Although QCOFDFT shares the same goals as the present QCDFT for quantum simulations at large scales, the two methods are very different in terms of the technical approach. While the QCDFT method is based on the Cauchy-Born rule (for the local region) and $\mathrm{QM} / \mathrm{MM}$ coupling (for the nonlocal region), QC-OFDFT relies on the finite element approximation for various meshes introduced in the method, including an atomic mesh, an electronic mesh, and a fine mesh. The quan- 
tum mechanical problem of OFDFT is solved on these meshes simultaneously. Elegant as the method is, its present formulation is limited to local approximations of the kinetic energy (i.e., the important kernel terms are not included), and it is not clear how the method could be extended either to nonlocal kinetic energy functionals for OFDFT or to a KohnSham DFT formulation. On the other hand, the present QCDFT does not suffer from the above constraints, and it could be extended to a Kohn-Sham DFT setting. Recent progress in sparse grid representation ${ }^{39}$ makes it possible to perform KS-DFT-based local QC calculations, and a $\mathrm{QM} / \mathrm{MM}$ method has been developed to treat nonlocal atoms with KS-DFT. ${ }^{5}$ KS-DFT is important for nonlocal QC because it offers superior accuracy and versatility than OFDFT in dealing with the nonlocal region for many material systems.

\section{COMPUTATIONAL DETAILS}

\section{A. Model setup}

Nanoindentation has now become a standard experimental technique for evaluating the mechanical properties of thin film materials and bulk materials in small volumes. ${ }^{40}$ As it can measure nanometer penetration length scales, nanoindentation is an indispensable tool to assess elastic moduli and hardness of materials. It also can be used to derive strainhardening exponents, fracture toughness, and viscoelastic properties of materials. ${ }^{41}$ Moreover, nanoindentation also provides an opportunity to explore and better understand the elastic limit and incipient plasticity of crystalline solids. ${ }^{42}$ For example, homogeneous nucleation of dislocations gives rise to the instability at the elastic limit of a perfect crystal. Exceeding the elastic limit can be manifested by a discontinuity in the load-displacement curve in a nanoindentation experiment. ${ }^{43-48}$ The onset of the discontinuity is an indication that the atomically localized deformation, such as dislocation nucleation, occurs beneath the indenter. This correlation has been well established from both experimental and computational perspectives. For example, an in situ experiment by Gouldstone et $a l .{ }^{49}$ using the Bragg-Nye bubble raft clearly demonstrated that homogeneous nucleation of dislocations corresponds to the discontinuity of the load-depth curve. MD simulations have led to greater insight into the atomistic mechanism of nanoindentation. ${ }^{50,51}$ In particular, several QC simulations have been carried out for nanoindentation in Al thin films. ${ }^{19,20,52-54}$ Tadmor et al. ${ }^{54}$ have used EAM-QC to study nanoindentation with a knifelike indenter with a pseudo-two-dimensional (2D) model. They observed the correspondence between the discontinuity in the loaddisplacement curve with the onset of plasticity. By using a much larger spherical indenter $(700 \mathrm{~nm})$, Knap et al. ${ }^{53}$ discovered that plasticity could occur without the corresponding discontinuity in the load-displacement curve. However, when the indenter size was reduced (to $70 \mathrm{~nm}$ ) the discontinuity reappeared. More recently, Hayes et al. ${ }^{19,20}$ have performed local OFDFT- and EAM-based QC calculations for nanoindentation of $\mathrm{Al}$ with a spherical indenter of $740 \mathrm{~nm}$ in radius. Using elastic stability criteria, they predicted the location of dislocation nucleation beneath the indenter and obtained dif-

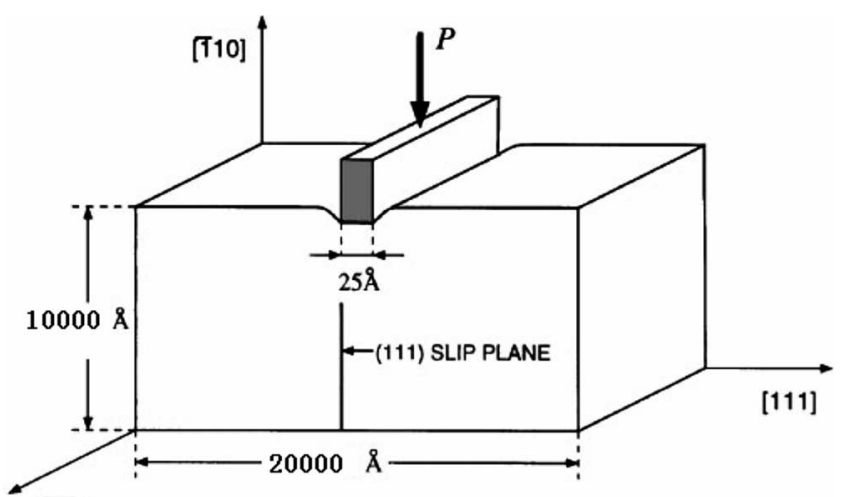

[112]

FIG. 2. Schematic representation of the nanoindentation of Al thin film: geometry and orientation.

ferent results from EAM and OFDFT local QC calculations. Since many QC simulations have been carried out for nanoindentation of $\mathrm{Al}$, it is not the purpose of the present paper (and we do not expect) to discover any new physics with QCDFT calculations. Instead, we use nanoindentation as an example to demonstrate the validity and the usefulness of the QCDFT method.

The present QCDFT approach is applied to nanoindentation of an $\mathrm{Al}$ thin film resting on a rigid substrate with a rigid knifelike indenter. The QC method is appropriate for the problem because it allows the modeling of system dimensions on the order of microns and thus minimizes the possibility of contaminating the results by the boundary conditions arising from small model sizes typically used in MD simulations. We chose this particular system for two reasons. First, there exists a good local pseudopotential ${ }^{18}$ and an excellent EAM potential ${ }^{55}$ for Al. Second, results from conventional EAM-based QC simulations can be compared to the present calculations. An ideal validation of the method would require a full-blown OFDFT atomistic simulation for nanoindentation, which is not yet attainable. The second best approach would be a conventional QC simulation with an excellent EAM potential that compares well to OFDFT in terms of critical materials properties relevant to nanoindentation. Our reasoning is that the conventional QC method has been well established; thus as long as the EAM potential used is reliable, then the EAM-QC results should be reliable as well. In this paper, we have rescaled the "force-matching" EAM potential of $\mathrm{Al}^{55}$ so that it matches precisely the OFDFT value of the lattice constant and bulk modulus of $\mathrm{Al}{ }^{32}$ Excellent results were obtained for perfect bulk Al calculations as a validation of the QM/MM coupling method used in the nonlocal QC region. ${ }^{5}$

The crystallographic orientation of the system is displayed in Fig. 2. The size of the entire system is $2 \mu \mathrm{m}$ $\times 1 \mu \mathrm{m} \times 4.9385 \AA$ along the [111] ( $x$ direction), the $[\overline{1} 10]$ ( $y$ direction), and the $[\overline{1} \overline{1} 2]$ ( $z$ direction), respectively. The system is periodic in the $z$ dimension, has Dirichlet boundary conditions in the other two directions, and contains over 60 million $\mathrm{Al}$ atoms - a size that is well beyond the reach of any full-blown brute-force quantum calculation. The thickness of the thin film is selected to be comparable to the typical dis- 
TABLE I. Elastic moduli, Poisson's ratio, lattice constant, (111) surface energy, and intrinsic stacking fault energy obtained by OFDFT and EAM calculations on bulk $\mathrm{Al}$, and the corresponding experimental values extrapolated to $T=0 \mathrm{~K}$.

\begin{tabular}{lcccc}
\hline \hline & \multicolumn{4}{c}{ Rescaled } \\
& OFDFT & EAM & Experiment & (Unit) \\
\hline Elastic modulus $C_{11}$ & 117.17 & 97.13 & 118.0 & $\mathrm{GPa}$ \\
Elastic modulus $C_{12}$ & 41.36 & 51.16 & 62.4 & $\mathrm{GPa}$ \\
Elastic modulus $C_{44}$ & 29.76 & 30.23 & 32.5 & $\mathrm{GPa}$ \\
Bulk modulus $E$ & 66.63 & 66.48 & 80.93 & $\mathrm{GPa}$ \\
Shear modulus $\mu$ & 33.01 & 27.33 & 30.62 & $\mathrm{GPa}$ \\
Poisson's ratio $\nu$ & 0.287 & 0.319 & 0.332 & \\
Lattice constant $a_{0}$ & 4.032 & 4.032 & 4.032 & $\AA$ \\
Surface energy $\gamma_{111}$ & 0.867 & 0.72 & $1.14-1.2$ & $\mathrm{~J} / \mathrm{m}^{2}$ \\
Stacking fault energy & 0.10 & 0.10 & $0.12-0.14$ & $\mathrm{~J} / \mathrm{m}^{2}$ \\
\hline \hline
\end{tabular}

location separation distance in well-annealed metals, which is of the order $1 \mu \mathrm{m}$. The unloaded system is a perfect single crystal similar to the experimental situation under the nanoindenter. The film is oriented so that the preferred slip system $\langle 110\rangle\{111\}$ is parallel to the indentation direction to facilitate dislocation nucleation. The indenter is a rigid flat punch of width $25 \AA$. We assume the perfect-stick boundary condition for the indenter so that the $\mathrm{Al}$ atoms in contact with it are not allowed to slip. The knifelike geometry of the indenter is dictated by the pseudo-2D nature of the QC model adopted. Three-dimensional QC models do exist and can be implemented in QCDFT. ${ }^{19,20,53}$ We chose to work with the pseudo-2D model in this example for its simplicity. The prefix pseudo is meant to emphasize that although the analysis is carried out in a 2D coordinate system, out-ofplane displacements are allowed and all atomistic calculations are three-dimensional. Within this setting only dislocations with line directions perpendicular to the $x y$ plane can be nucleated. The elastic moduli of $C_{12}, C_{44}, C_{11}$ of $\mathrm{Al}$ are computed from three deformation modes, including hydrostatic, volume-conserving tetragonal and volume-conserving rhombohedral deformations. The shear modulus $\mu$ and Poisson's ratio $\nu$ are computed from the elastic moduli by a Voigt average: $\mu=\left(C_{11}-C_{12}+3 C_{44}\right) / 5$ and $\nu=\frac{C_{11}+4 C_{12}-2 C_{44}}{2\left(2 C_{11}+3 C_{12}+C_{44}\right)}$. The values are listed in Table I.

\section{B. Loading procedure}

The simulation is performed quasistatically with a displacement control where the indentation depth $(d)$ is increased by $0.2 \AA$ at each loading step. We also tried a smaller loading step of $0.1 \AA$ and obtained essentially the same results. Because OFDFT calculations are still much more expensive than EAM, we use EAM-based QC to relax the system for most of the loading steps. For load $d=0$, the QCDFT calculation is performed to account for surface relaxations. From the resultant configuration, the depth of the indenter $d$ is increased to $0.2 \AA$, again relaxed by QCDFT. After that, the calculations are done solely by EAM-QC ex- cept for the loading steps at $d=1.8,3.8,9.2 \AA$, when the corresponding EAM configurations are further relaxed by QCDFT. The onset of plasticity occurs at $d=9.4 \AA$. We increased the indenter depth of $0.2 \AA$ from the relaxed QCDFT configuration at $d=9.2 \AA$, and then performed a QCDFT calculation to obtain the final structure at $d$ $=9.4 \AA$ A Such a simulation strategy is justified based on two considerations: (i) An earlier nanoindentation study of the same $\mathrm{Al}$ surface found that the onset of plasticity occurred at a smaller load with EAM-based local QC calculations comparing to OFDFT calculations. ${ }^{20}$ The result was obtained by a local elastic stability analysis with EAM and OFDFT calculations of energetics and stress. The result suggests that we will not miss the onset of plasticity with the present loading procedure by performing EAM-QC relaxations preceding QCDFT. (ii) Before the onset of plasticity, the loaddisplacement response is essentially linear with the slope determined by the elastic properties of the material. In other words, two QCDFT data points would be sufficient to obtain the correct linear part of the curve. Moreover, the fact that the EAM potential used in this study yields rather similar elastic constants to those from OFDFT suggests that the mixed EAM/OFDFT relaxation should not introduce large errors in the results.

\section{Computation parameters}

In Fig. 1, we present a schematic diagram illustrating the partition of domains for a QCDFT simulation of nanoindentation. The system shown in the diagram contains 1420 nonlocal repatoms, 736 local repatoms and 1539 finite elements, and is periodic along the $z$ direction. The system corresponds to the deformation state close to onset of incipient plasticity. The top surface is allowed to relax during the calculations while the other three surfaces of the sample are held fixed.

The parameters of the density-dependent kernel are chosen from Ref. 10 and $\mathrm{Al}$ ions are represented by the Goodwin-Needs-Heine local pseudopotential. ${ }^{18}$ The high kinetic energy cutoff for the plane-wave basis of $1600 \mathrm{eV}$ is used to ensure the convergence of the charge density. For the nonlocal calculation, the grid density for the volume $\Omega^{\mathrm{I}}$ is 5 grid points per $\AA$. The $\Omega^{\mathrm{I}}$ box goes beyond the nonlocal region by $8 \AA$ in $\pm x$ and $\pm y$ directions so that $\rho^{\mathrm{I}}$ decays to zero at the boundary of $\Omega^{\mathrm{I}}$. All parameters that are relevant to $\mathrm{QM} / \mathrm{MM}$ coupling have been tested in our previous study. ${ }^{5}$ The relaxation of all repatoms is performed by a conjugate gradient method until the maximum force on any repatom is less than $0.03 \mathrm{eV} / \AA$.

At beginning of the simulation, the number of nonlocal repatoms is rather small, $\sim 80$. As the load increases, the material deforms. When the variation of the deformation gradient between neighboring finite elements reaches 0.15 , the mesh is refined, and the number of repatoms grows. Close to the onset of plasticity, the number of nonlocal DFT atoms reaches 1420. This procedure of automatic mesh adaption is well documented in QC literature ${ }^{1,2}$ and our tests on EAM-QC have shown that 0.15 is sufficient to give converged results.

In order to validate the QCDFT method, we performed EAM-QC calculations of the nanoindentation with the same 


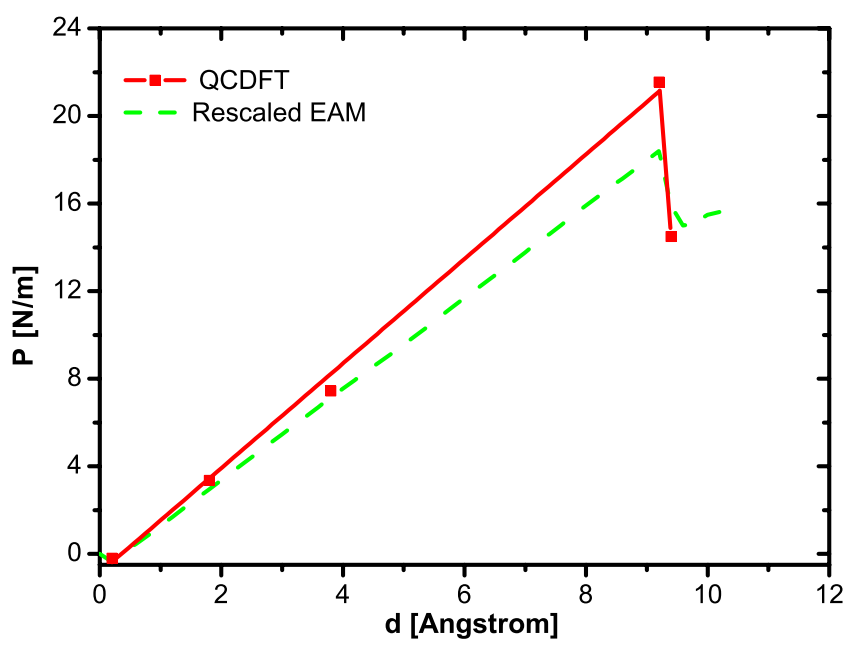

FIG. 3. (Color online) Load-displacement curve for nanoindentation of an Al thin film with a rigid rectangular indenter: with QCDFT (solid line) and rescaled EAM-QC (dashed line). The squares are actual QCDFT data points and the solid line is the best fit to the data points. All EAM-QC data points are on the dashed line.

loading steps. We also calculated some relevant materials properties using the rescaled EAM and OFDFT method for bulk $\mathrm{Al}$ - these are standard atomistic calculations, not multiscale QC or QCDFT simulations. Since what distinguishes EAM-QC from QCDFT is in their underlying energetics, EAM vs OFDFT, the EAM and OFDFT calculations for the relevant material properties could shed light on the different results obtained from the EAM-QC and QCDFT simulations on nanoindentation. The results of EAM and OFDFT calcu- lations along with experimental values extrapolated to $T$ $=0 \mathrm{~K}^{55}$ are listed in Table I.

\section{RESULTS AND ANALYSIS}

The load-displacement curve is the typical observable for nanoindentation, and is widely used in both experiment and theory, often serving as a link between the two. In particular, it is conventional to identify the onset of plasticity with the first jump in the load-displacement curve during indentation. ${ }^{20,42,46-48,52-54,56}$ In the present work, the loads are given in $\mathrm{N} / \mathrm{m}$, normalized by the length of the indenter in the out-of-plane direction.

Let us first discuss the QC results with the rescaled EAM potential. The load-displacement $(P-d)$ curve shows a linear relation followed by a discrete drop at $d=9.4 \AA$, shown by the dashed line in Fig. 3. The drop corresponds to the homogeneous nucleation of dislocations beneath the indenter-the onset of plasticity. A pair of straight edge dislocations is nucleated at $x= \pm 13 \AA$, and $y=-49 \AA$. In Fig. 4, we present the out-of-plane (or screw) displacement $u_{z}$ of the nonlocal repatoms. The nonzero screw displacement of edge dislocations suggests that each dislocation is dissociated into two $1 / 6\langle 112\rangle$ Shockley partials bound by a stacking fault with a width of about $14 \AA$. An earlier EAM-QC calculation ${ }^{54}$ which has the same geometry as the present model but with a thinner sample (the thickness was ten times smaller than the present case) yields a separation distance of $13.5 \AA$. The activated slip planes are those $\{111\}$ planes that are adjacent to the side surfaces of the indenter. The linear relation in the $P-d$ curve is due to (i) the elastic response of the material before the onset of plasticity and (ii) the particular choice of
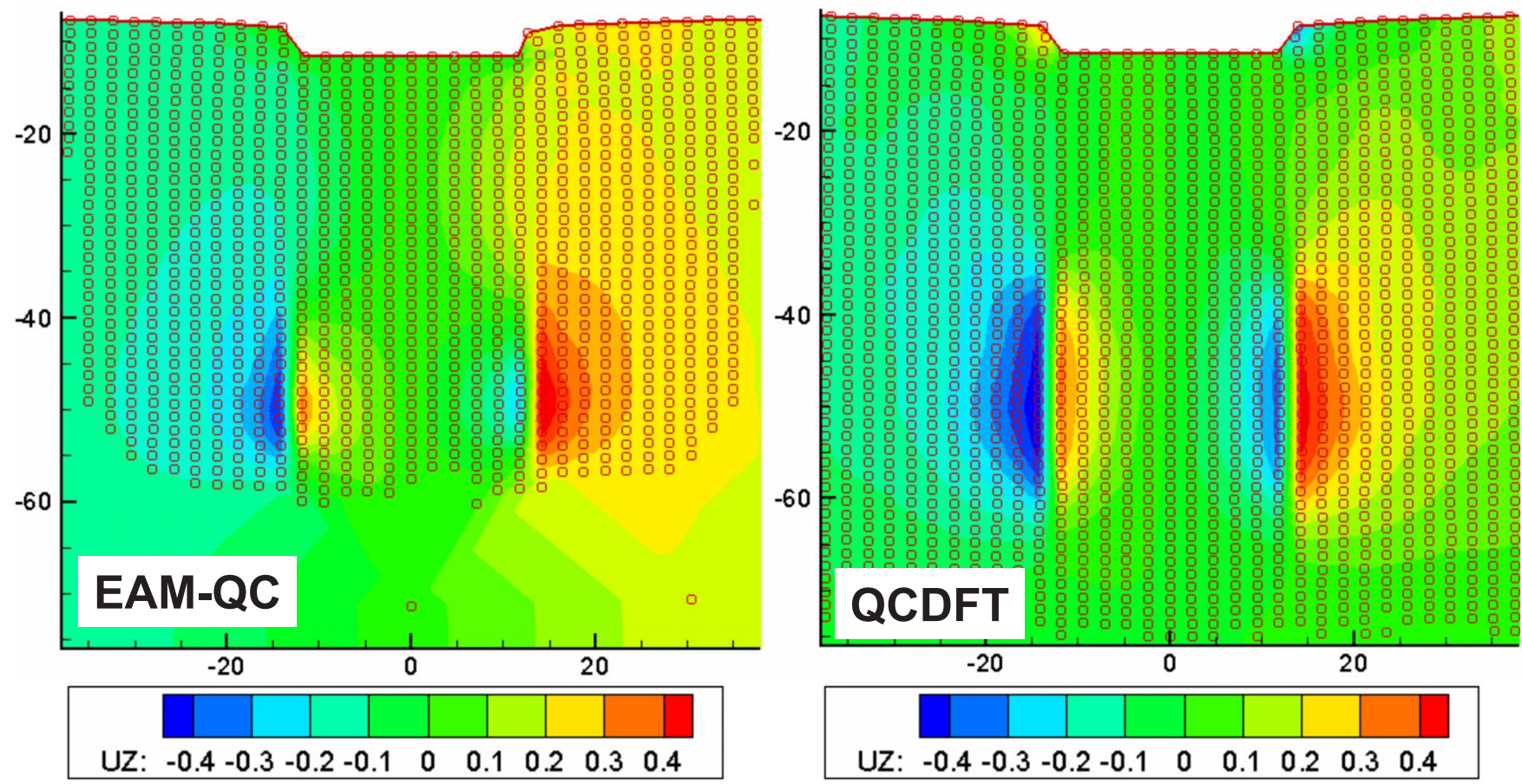

FIG. 4. (Color online) The out-of-plane displacement $u_{z}$ obtained from the rescaled EAM-QC (left) and QCDFT (right) calculations. The circles represent the repatoms and the displacement ranges from -0.4 to $0.4 \AA$. 
the rectangular indenter; a spherical indenter would have given rise to a parabolic $P-d$ curve. ${ }^{20,48}$ The slope for the linear part of the curve is $20.8 \mathrm{GPa}$, which is less than the shear modulus and $C_{44}$. The critical load, $P_{\text {cr }}$, for homogeneous dislocation nucleation is $18.4 \mathrm{~N} / \mathrm{m}$, corresponding to a hardness of 7.3 GPa. Earlier EAM-QC calculations predicted the hardness to be $9.8 \mathrm{Gpa} .{ }^{54}$ The drop in applied load due to the nucleation of dislocations is $\Delta P=3.4 \mathrm{~N} / \mathrm{m}$. The value of $\Delta P$ from the previous EAM-QC calculation is $10 \mathrm{~N} / \mathrm{m},{ }^{54}$ which is three times of the present result. The discrepancy of $\Delta P$ is mainly due to the different sample sizes used in the two calculations ${ }^{57}$ indicating the importance of simulations at length scales relevant to experiments.

For QCDFT calculations, the load-displacement curve shows a linear relation up to a depth of $9.2 \AA$, followed by a drop at $d=9.4 \AA$, shown by the solid line in Fig. 3. The slope of initial linear part of the load-displacement curve is 23.9 GPa, rather close to the corresponding EAM value. The maximum load in linear region is $P_{\mathrm{cr}}=21.4 \mathrm{~N} / \mathrm{m}$, corresponding to a hardness of $8.6 \mathrm{GPa}$. The fact that OFDFT predicts a larger $P_{\text {cr }}$ than EAM is consistent with the results of Hayes et al. ${ }^{20}$ using local QC simulations for the same Al surface. A pair of edge dislocations is nucleated at $x$ $= \pm 13 \AA$, and $y=-50 \AA$. The partial separation distance is about $19 \AA$, larger than the corresponding EAM value. The drop in the applied load due to dislocation nucleation is 7.8 $\mathrm{N} / \mathrm{m}$, which is more than twice the corresponding EAM value. The large difference in $\Delta P$ between QCDFT and EAM-QC is interesting. It may suggest that although OFDFT and EAM produce rather similar results before the onset of plasticity, they differ significantly in describing certain aspects of defect properties. In particular, although both methods predict almost the same location for dislocation nucleation, they yield sizeable differences in partial dislocation width and $\Delta P$. This result justifies the use of more accurate quantum simulations such as KS-DFT for the nonlocal region where defects are present. Overall, we find that QCDFT gives very reasonable results comparing to the conventional EAM-QC. Although more validations are underway, we are optimistic that the QCDFT method is indeed reliable and offers a new route for quantum simulation of materials at large length scales.

\section{CONCLUSION}

In summary, we present a concurrent multiscale method that makes it possible to simulate multimillion atoms based on density-functional theory. The method-QCDFT-is formulated within the framework of the QC method, with OFDFT as its sole input, i.e., there is only one underlying energy functional (OFDFT) involved. Full-blown OFDFT and OFDFT-based elasticity theory are the two limiting cases corresponding to a fully nonlocal or a fully local version of QCDFT. The QC ghost force at the local-nonlocal interface is corrected by a dead load approximation. The QCDFT method is applied for a nanoindentation study of an Al thin film. The QCDFT results are validated by comparing against conventional QC with an OFDFT-refined EAM potential. The results suggest that QCDFT is an excellent method for quantum simulation of materials properties at length scales relevant to experiments.

\section{ACKNOWLEDGMENTS}

The work at California State University Northridge was supported by NSF PREM Grant No. DMR-0611562 and DOE SciDAC Grant No. DE-FC02-06ER25791. The work at Princeton was supported by the NSF (E.A.C.) and NDSEG foundation (L.H.). *ganglu@csun.edu

${ }^{1}$ E. B. Tadmor, M. Ortiz, and R. Phillips, Philos. Mag. A 73, 1529 (1996).

${ }^{2}$ V. B. Shenoy, R. Miller, E. B. Tadmor, D. Rodney, R. Phillips, and M. Ortiz, J. Mech. Phys. Solids 47, 611 (1999).

${ }^{3} \mathrm{G}$. Lu and E. Kaxiras, in Handbook of Theoretical and Computational Nanotechnology, edited by M. Rieth and W. Schmmers (American Scientific, Stevenson Ranch, CA, 2004), Chap. 22.

${ }^{4}$ O. C. Zienkiewicz and R. L. Taylor, The Finite Element Method: The Basis (Butterworth, Oxford, 2000), Vol. 1, p. 23.

${ }^{5}$ X. Zhang and G. Lu, Phys. Rev. B 76, 245111 (2007).

${ }^{6}$ Y. A. Wang and E. A. Carter, in Theoretical Methods in Condensed Phase Chemistry, edited by S. D. Schwartz (Kluwer, Dordrecht, 2000), Chap. 5.

${ }^{7}$ S. C. Watson and E. A. Carter, Comput. Phys. Commun. 128, 67 (2000).

${ }^{8}$ V. Lignères and E. A. Carter, in Handbook of Materials Modeling, edited by S. Yip (Springer, Dordrecht, The Netherlands), pp. 137-148.

${ }^{9}$ L. W. Wang and M. P. Teter, Phys. Rev. B 45, 13196 (1992).

${ }^{10}$ Y. A. Wang, N. Govind, and E. A. Carter, Phys. Rev. B 60,
16350 (1999).

${ }^{11}$ P. Garcia-Gonzalez, J. E. Alvarellos, and E. Chacon, Phys. Rev. B 53, 9509 (1996).

${ }^{12}$ L. Hung and E. A. Carter (unpublished).

${ }^{13}$ L. H. Thomas, Proc. Cambridge Philos. Soc. 23, 542 (1926).

${ }^{14}$ E. Fermi, Z. Phys. 48, 73 (1928).

${ }^{15}$ C. F. V. Weizsäcker, Z. Phys. 96, 431 (1935).

${ }^{16}$ J. Lindhard, K. Dan. Vidensk. Selsk. Mat. Fys. Medd. 28, 8 (1954).

${ }^{17}$ Y. A. Wang, N. Govind, and E. A. Carter, Phys. Rev. B 64, 089903(E) (2001).

${ }^{18}$ L. Goodwin, R. J. Needs, and V. Heine, J. Phys.: Condens. Matter 2, 351 (1990).

${ }^{19}$ R. L. Hayes, G. Ho, M. Ortiz, and E. A. Carter, Philos. Mag. 86, 2343 (2006).

${ }^{20}$ R. L. Hayes, M. Fago, M. Ortiz, and E. A. Carter, Multiscale Model. Simul. 4, 359 (2005).

${ }^{21}$ J. P. Perdew and A. Zunger, Phys. Rev. B 23, 5048 (1981).

${ }^{22}$ D. M. Ceperley and B. J. Alder, Phys. Rev. Lett. 45, 566 (1980).

${ }^{23}$ J. C. Slater, Phys. Rev. 81, 385 (1951).

${ }^{24}$ P. P. Ewald, Ann. Phys. 64, 253 (1921). 
${ }^{25}$ A. Y. Toukmaji and J. A. Board, Comput. Phys. Commun. 95, 73 (1996).

${ }^{26}$ N. Karasawa and W. A. Goddard III, J. Phys. Chem. 93, 7320 (1989).

${ }^{27}$ H. Lin and D. G. Truhlar, Theor. Chem. Acc. 117, 185 (2007).

${ }^{28}$ T. Mordasini and W. Thiel, Chimia 52, 288 (1998).

${ }^{29}$ T. K. Woo P. M. Margl, L. Deng, L. Cavallo, and T. Ziegler, ACS Symp. Ser. 721, 173 (1999).

${ }^{30} \mathrm{P}$. Sherwood, in Modern Methods and Algorithms of Quantum Chemistry, edited by J. Grotendorst (NIC, Princeton, 2000), Vol. 3, p. 285.

${ }^{31}$ J. Gao and D. G. Truhlar, Annu. Rev. Phys. Chem. 53, 467 (2002).

${ }^{32}$ N. Choly, G. Lu, W. E, and E. Kaxiras, Phys. Rev. B 71, 094101 (2005).

${ }^{33}$ G. S. Smith, E. B. Tadmor, N. Bernstein, and E. Kaxiras, Acta Mater. 49, 4089 (2001).

${ }^{34}$ E. B. Tadmor, G. S. Smith, N. Bernstein, and E. Kaxiras, Phys. Rev. B 59, 235 (1999).

${ }^{35}$ E. B. Tadmor, U. V. Waghmare, G. S. Smith, and E. Kaxiras, Acta Mater. 50, 2989 (2002).

${ }^{36}$ G. Lu, E. B. Tadmor, and E. Kaxiras, Phys. Rev. B 73, 024108 (2006).

${ }^{37}$ Q. Peng, E. B. Tadmor, and G. Lu (unpublished).

${ }^{38}$ V. Gavini, K. Bhattacharya, and M. Ortiz, J. Mech. Phys. Solids 55, 697 (2007).

${ }^{39}$ C. J. García-Cervera, W. Ren, J. Lu, and W. E., Comm. Comp. Phys. 4, 1025 (2008).

${ }^{40}$ W. D. Nix, Metall. Trans. A 20, 2217 (1989).

${ }^{41}$ A. C. Fischer-Cripps, Nanoindentation, 2nd ed. (Springer-
Verlag, New York, 2004), p. 1.

${ }^{42}$ T. Zhu, J. Li, J. Van Vliet, S. Ogata, S. Yip, and S. Suresh, J. Mech. Phys. Solids 52, 691 (2004).

${ }^{43}$ T. F. Page, W. C. Oliver, and C. J. McHargue, J. Mater. Res. 7, 450 (1992).

${ }^{44}$ W. C. Oliver and G. M. Pharr, J. Mater. Res. 7, 1564 (1992).

${ }^{45}$ W. W. Gerberich, J. C. Nelson, E. T. Lilleodden, P. Anderson, and J. T. Wyrobek, Acta Mater. 44, 3585 (1996).

${ }^{46}$ S. G. Corcoran, R. J. Colton, E. T. Lilleodden, and W. W. Gerberich, Phys. Rev. B 55, R16057 (1997).

${ }^{47}$ S. Suresh, T. G. Nieh, and B. W. Choi, Scr. Mater. 41, 951 (1999).

${ }^{48}$ A. Gouldstone, H. J. Koh, K. Y. Zeng, A. E. Giannakopoulos, and S. Suresh, Acta Mater. 48, 2277 (2000).

${ }^{49}$ A. Gouldstone, K. J. Van Vliet, and S. Suresh, Nature (London) 411, 656 (2001).

${ }^{50}$ C. L. Kelchner, S. J. Plimpton, and J. C. Hamilton, Phys. Rev. B 58, 11085 (1998).

${ }^{51}$ J. A. Zimmerman, C. L. Kelchner, P. A. Klein, J. C. Hamilton, and S. M. Foiles, Phys. Rev. Lett. 87, 165507 (2001).

${ }^{52}$ V. B. Shenoy, R. Phillips, and E. B. Tadmor, J. Mech. Phys. Solids 48, 649 (2000).

${ }^{53}$ J. Knap and M. Ortiz, Phys. Rev. Lett. 90, 226102 (2003).

${ }^{54}$ E. B. Tadmor, R. Miller, and R. Phillips, J. Mater. Res. 14, 2233 (1999).

${ }^{55}$ F. Ercolessi and J. B. Adams, Europhys. Lett. 26, 583 (1994).

${ }^{56}$ A. Gouldstone, N. Chollacoop, M. Dao, J. Li, A. M. Minor, and Y. Shen, Acta Mater. 55, 4015 (2007).

${ }^{57}$ Using the same sample size as in Ref. 54, we found $\Delta P$ $=9.02 \mathrm{~N} / \mathrm{m}$, which is very close to the value reported in Ref. 54. 